\title{
Derivation of effective macroscopic Stokes-Cahn-Hilliard equations for periodic immiscible flows in porous media
}

\author{
Markus Schmuck ${ }^{1,2}$, Marc Pradas ${ }^{1}$, Grigorios A. Pavliotis ${ }^{2}$ \\ and Serafim Kalliadasis ${ }^{1}$ \\ ${ }^{1}$ Department of Chemical Engineering, Imperial College London, South Kensington \\ Campus, SW7 2AZ London, UK \\ ${ }^{2}$ Department of Mathematics, Imperial College London, South Kensington Campus, \\ SW7 2AZ London, UK \\ E-mail: \\ M.Schmuck@hw.ac.uk, m.pradas@imperial .ac.uk, g.pavliotis@imperial.ac.uk, \\ and s.kalliadasis@imperial.ac.uk
}

\begin{abstract}
Using thermodynamic and variational principles we examine a basic phase field model for a mixture of two incompressible fluids in strongly perforated domains. With the help of the multiple scale method with drift and our recently introduced splitting strategy for Ginzburg-Landau/Cahn-Hilliard-type equations [42, we rigorously derive an effective macroscopic phase field formulation under the assumption of periodic flow and a sufficiently large Péclet number. As for classical convection-diffusion problems, we obtain systematically diffusion-dispersion relations (including Taylor-Aris-dispersion). Our results also provide a convenient analytical and computational framework to macroscopically track interfaces in porous media. In view of the well-known versatility of phase field models, our study proposes a promising model for many engineering and scientific applications such as multiphase flows in porous media, microfluidics, and fuel cells.
\end{abstract}

Submitted to: Nonlinearity

$\ddagger$ Present address: School of Mathematical and Computer Sciences and the Maxwell Institute for Mathematical Sciences, Heriot-Watt University, EH14 4AS, Edinburgh, UK 


\section{Introduction}

Fluid mixtures are ubiquitous in many scientific and engineering applications. The dynamics of phase interfaces between fluids plays a central role in rheology and hydrodynamics [9, 12, 17, 22, 34]. A recent attempt of a systematic extension towards non-equilibrium two-phase systems is [38], where the authors discuss the concept of local thermodynamic equilibrium of a Gibbs interface in order to relax the global thermodynamic equilibrium assumption. In [24, it is shown that the Cahn-Hilliard or diffuse interface formulation of a quasi-compressible binary fluid mixture allows for topological changes of the interface. Also of increasing interest is the mathematical and physical understanding of wetting using diffuse interface formulations [46, 45] as well as wetting in the presence of complexities such as electric fields [25, 16].

The study of flows in porous media is a delicate multiscale problem. This is evident, for instance, by the fact, that the full problem without any approximations is not computationally tractable with the present computational power [2, 20]. Also, from an empirical perspective the consideration of the full multiscale problem is very challenging due to the difficulty of obtaining detailed information about the pore geometry. These empirical and computational restrictions strongly call for systematic and reliable approximations which capture the essential physics and elementary dynamic characteristics of the full problem in an averaged sense. A very common and intuitive strategy is volume averaging [36, 50]. The method of moments [4, 10] and multiple scale expansions [7, 33, 13] have been used in this context. The latter method is more systematic and reliable since it allows for a rigorous mathematical verification. The volume averaging strategy still lacks a consistent and generally accepted treatment of nonlinear terms. Therefore, the multiscale expansion strategy is used as a basis for the theoretical developments in the present study.

The celebrated works in [4, 10, 48] initiated an increasing interest in the understanding of hydrodynamic dispersion on the spreading of tracer particles transported by flow, with numerous applications, from transport of contaminants in rivers to chromatogaphy. In [37, it is shown that the multiscale expansion strategy allows to recover the dispersion relation found in [10]. The study of multiphase flows in porous media is considerably more complex; see e.g. the comprehensive review in [2] which still serves as a basis for several studies in the field. The central idea for many approaches to multiphase flows is to extend Darcy's law to multiple phases. With the help of Marle's averaging method [26] and a diffuse interface model, effective two-phase flow equations are presented in [31, 32]. In [5], Atkin and Craine even

present constitutive theories for a binary mixture of fluids and a porous elastic solid. A combination of the homogenization method and a multiphase extension of Darcy's law as a description of multiphase flows in porous media is applied in the articles [8, 43], for instance.

An application of increasing importance for a renewable energy infrastructure are fuel cells [35]. This article combines the complex multiphase interactions with the help 
of the Cahn-Hilliard phase field method and a total free energy characterizing the fuel cell. An upscaling of the full thermodynamic model proposed in [35] is obviously very involved due to complex interactions over different scales. In this context, an upscaled macroscopic description of a simplified (i.e., no fluid flow and periodic catalyst layer) is derived in [41, 40].

Consider the total energy density for an interface between two phases,

$$
\left.e(\mathbf{x}(\mathbf{X}, t), t):=\frac{1}{2}\left|\frac{\partial \mathbf{x}(\mathbf{X}, t)}{\partial t}\right|^{2}-\frac{\lambda}{2} \mid \nabla_{\mathbf{x}} \phi(\mathbf{x}(\mathbf{X}, t), t)\right)\left.\right|^{2}-\frac{\lambda}{2} F(\phi(\mathbf{x}(\mathbf{X}, t), t)),
$$

where $\phi$ is a conserved order-parameter that evolves between different liquid phases represented as the minima of a homogeneous free energy $F$. The parameter $\lambda$ represents the surface tension effect, i.e. $\lambda \propto$ (surface tension) $\times$ (capillary width) $=\sigma \eta$. The variable $\mathbf{X}$ stands for the Lagrangian (initial) material coordinate and $\mathbf{x}(\mathbf{X}, t)$ represents the Eulerian (reference) coordinate. Our derivation (Section 4) is valid for general free energies $F$ and uses the method of an asymptotic multiscale expansion with drift [3]. Furthermore, we establish the wellposedness (Theorem 3.5) of the upscaled/homogenized equations for polynomial free energies of the following form [49]:

Assumption (PF): The free energy densities $F$ in (1) are polynomials of order $2 r-1$, i.e.,

$$
f(u)=\sum_{i=1}^{2 r-1} a_{i} u^{i}, \quad r \in \mathbb{N}, \quad r \geq 2
$$

with $f(u)=F^{\prime}(u)$ vanishing at $u=0$,

$$
F(u)=\sum_{i=2}^{2 r} b_{i} u^{i}, \quad i b_{i}=a_{i-1}, \quad 2 \leq i \leq 2 r,
$$

where the leading coefficient of both $F$ and $f$ is positive, i.e., $a_{2 r-1}=2 r b_{2 r}>0$.

Remark 1.1 (Double-well potential) Free energies F satisfying the Assumption (PF) form a general class which also includes the double-well potential for $r=2$ with $f(u)=-\alpha u+\beta u^{3}, \alpha, \beta>0$, for which $\left(\eta_{4}\right.$ is called the convective Cahn-Hilliard equation. We note that the double-well is scaled by $\frac{1}{4 \eta^{2}}$, i.e., $F(u)=\frac{1}{4 \eta^{2}}\left(u^{2}-1\right)^{2}$ such that one recovers the Hele-Shaw problem in the limit $\eta \rightarrow 0$ [19, 22].

The last two terms in (1) form the well-known density of the CahnHilliard/Ginzburg-Landau phase field formulation adapted to the flow map $\mathbf{x}(\mathbf{X}, t)$ defined by

$$
\left\{\begin{array}{l}
\frac{\partial \mathbf{x}}{\partial t}=\mathbf{u}(\mathbf{x}(\mathbf{X}, t), t) \\
\mathbf{x}(\mathbf{X}, 0)=\mathbf{X}
\end{array}\right.
$$


The first term in (1) is the kinetic energy, which accounts for the fluid flow of incompressible materials, i.e.,

$$
\left\{\begin{array}{l}
\frac{\partial \mathbf{u}}{\partial t}+(\mathbf{u} \cdot \nabla) \mathbf{u}-\mu \Delta \mathbf{u}+\nabla p=\boldsymbol{\eta} \\
\operatorname{div} \mathbf{u}=0
\end{array}\right.
$$

where we additionally added the second order term multiplied by the viscosity $\mu$. The variable $\boldsymbol{\eta}$ is a driving force acting on the fluid. We are interested in the mixture of two incompressible and immiscible fluids of the same viscosity $\mu$. Hence, we can employ generic free energies (1) showing a double-well form as is the case often in applications, e.g. [51].

Suppose that $\Omega \subset \mathbb{R}^{d}$, with $d>0$ the dimension of space, denotes the domain which is initially occupied by the fluid. Then, we can define for an arbitrary length of time $T>0$ the total energy by

$$
E(\mathbf{x}):=\int_{0}^{T} \int_{\Omega} e(\mathbf{x}(\mathbf{X}, t), t) d \mathbf{X} d t
$$

The energy (6) combines an action functional for the flow map $\mathbf{x}(\mathbf{X}, t)$ and a free energy for the order parameter $\phi$. This combination of mechanical and thermodynamic energies seems to go back to [14, 15, 21, 22, 24]. Subsequently, we will focus on quasi-stationary, i.e., $\mathbf{u}_{t}=\mathbf{0}$ and $\boldsymbol{\eta} \neq \mathbf{0}$, and low-Reynolds number flows such that we can neglect the nonlinear term $(\mathbf{u} \cdot \nabla) \mathbf{u}$. Then, classical ideas from the calculus of variations [47] and the theory of gradient flows together with an imposed wetting boundary condition $\int_{\partial \Omega} g(\mathbf{x}) d o(\mathbf{x})$ for $g(\mathbf{x}) \in H^{3 / 2}(\partial \Omega)$ lead to the following set of equations

$$
\text { (Homogeneous case) } \begin{cases}-\mu \Delta \mathbf{u}+\nabla p=\boldsymbol{\eta} & \text { in } \Omega_{T}, \\ \operatorname{div} \mathbf{u}=0 & \text { in } \Omega_{T}, \\ \mathbf{u}=\mathbf{0} & \text { on } \partial \Omega_{T}, \\ \frac{\partial \phi}{\partial t}+\operatorname{Pe}(\mathbf{u} \cdot \nabla) \phi=\lambda \operatorname{div}(\nabla(f(\phi)-\Delta \phi)) & \text { in } \Omega_{T}, \\ \nabla_{n} \phi:=\mathbf{n} \cdot \nabla \phi=g(\mathbf{x}) & \text { on } \partial \Omega_{T}, \\ \nabla_{n} \Delta \phi=0 & \text { on } \partial \Omega_{T}, \\ \phi(\mathbf{x}, 0)=h(\mathbf{x}) & \text { on } \Omega,\end{cases}
$$

where $\left.\Omega_{T}:=\Omega \times\right] 0, T\left[, \partial \Omega_{T}:=\partial \Omega^{1} \times\right] 0, T[, \lambda$ represents the elastic relaxation time of the system, and the driving force $\boldsymbol{\eta}$ accounts for the elastic energy [22]

$$
\boldsymbol{\eta}=-\gamma \operatorname{div}(\nabla \phi \otimes \nabla \phi)
$$

where $\gamma$ corresponds to the surface tension [23]. As in [1], we will set $\gamma=\lambda$ for simplicity. The dimensionless parameter $\mathrm{Pe}:=\frac{k \tau L \mathrm{U}}{D}$ is the Péclet number for a reference fluid velocity $\mathrm{U}:=|\mathbf{u}|, L$ is the characteristic length of the porous medium, and the diffusion constant $D=k \tau M$ obtained from the mobility via Einstein's relation for the 
temperature $\tau$ and the Bolzmann constant $k$. We note that the immiscible flow equations can immediately be written for the full incompressible Navier-Stokes equations as in [22]. Our restriction to the Stokes equation is motivated here by the fact that such flows turn into Darcy's law in porous media [11, 18].

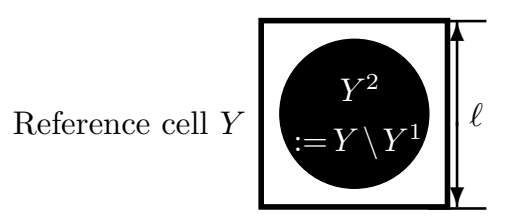

Periodic covering by cells $Y$

Homogenous approximation

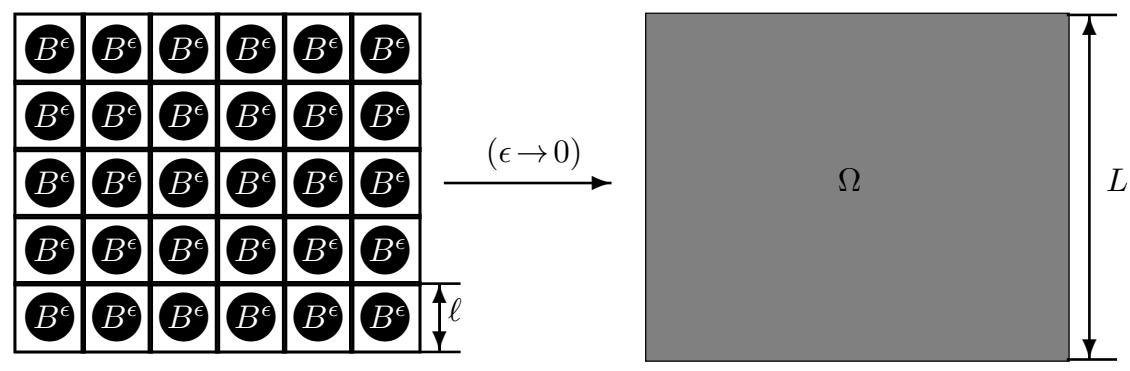

Figure 1. Left: Porous medium $\Omega^{\epsilon}:=\Omega \backslash B^{\epsilon}$ as a periodic covering of reference cells $Y:=[0, \ell]^{d}$. Top: Definition of the reference cell $Y=Y^{1} \cup Y^{2}$ with $\ell=1$. Right: The "homogenization limit" $\epsilon:=\frac{\ell}{L} \rightarrow 0$ scales the perforated domain such that perforations become invisible in the macroscale.

The main objective of our study is the derivation of effective macroscopic equations describing (7) in the case of perforated domains $\Omega^{\epsilon} \subset \mathbb{R}^{d}$ instead of a homogeneous $\Omega \subset \mathbb{R}^{d}$. A useful and reasonable approach is to represent a porous medium $\Omega=\Omega^{\epsilon} \cup B^{\epsilon}$ periodically with pore space $\Omega^{\epsilon}$ and solid phase $B^{\epsilon}$. The arising interface $\partial \Omega^{\epsilon} \cap \partial B^{\epsilon}$ is denoted by $I^{\epsilon}$. As usual, the dimensionless variable $\epsilon>0$ defines the heterogeneity $\epsilon=\frac{\ell}{L}$ where $\ell$ represents the characteristic pore size and $L$ is the characteristic length of the porous medium, see Figure 1 . The porous medium is defined by a periodic coverage of a reference cell $Y:=\left[0, \ell_{1}\right] \times\left[0, \ell_{2}\right] \times \cdots \times\left[0, \ell_{d}\right], \ell_{i} \in \mathbb{R}, i=1, \ldots, d$, which represents a single, characteristic pore. The periodicity assumption allows, by passing to the limit $\epsilon \rightarrow 0$ (see Figure 1) for the derivation effective macroscopic porous media equations. The pore and the solid phase of the medium are defined as usual by,

$$
\Omega^{\epsilon}:=\bigcup_{\mathbf{z} \in \mathbb{Z}^{d}} \epsilon\left(Y^{1}+\mathbf{z}\right) \cap \Omega, \quad B^{\epsilon}:=\bigcup_{\mathbf{z} \in \mathbb{Z}^{d}} \epsilon\left(Y^{2}+\mathbf{z}\right) \cap \Omega=\Omega \backslash \Omega^{\epsilon},
$$

where the subsets $Y^{1}, Y^{2} \subset Y$ are defined such that $\Omega^{\epsilon}$ is a connected set. More precisely, $Y^{1}$ denotes the pore phase (e.g. liquid or gas phase in wetting problems), see Figure 1. 
These definitions allow us to rewrite (7) by the following microscopic formulation

$$
\text { (Porous case }) \begin{cases}-\epsilon^{2} \mu \Delta \mathbf{u}_{\epsilon}+\nabla p_{\epsilon}=-\gamma \operatorname{div}\left(\nabla \phi_{\epsilon} \otimes \nabla \phi_{\epsilon}\right) & \text { in } \Omega_{T}^{\epsilon}, \\ \operatorname{div} \mathbf{u}_{\epsilon}=0 & \text { in } \Omega_{T}^{\epsilon}, \\ \mathbf{u}_{\epsilon}=\mathbf{0} & \text { on } I_{T}^{\epsilon}, \\ \frac{\partial}{\partial t} \phi_{\epsilon}+\operatorname{Pe}\left(\mathbf{u}_{\epsilon} \cdot \nabla\right) \phi_{\epsilon}=\lambda \operatorname{div}\left(\nabla\left(f\left(\phi_{\epsilon}\right)-\Delta \phi_{\epsilon}\right)\right) & \text { in } \Omega_{T}^{\epsilon}, \\ \nabla_{n} \phi_{\epsilon}:=\mathbf{n} \cdot \nabla \phi_{\epsilon}=g_{\epsilon}(\mathbf{x}):=g(\mathbf{x} / \epsilon) & \text { on } I_{T}^{\epsilon}, \\ \nabla_{n} \Delta \phi_{\epsilon}=0 & \text { on } I_{T}^{\epsilon}, \\ \phi_{\epsilon}(\cdot, 0)=\psi(\cdot) & \text { on } \Omega^{\epsilon},\end{cases}
$$

where $\left.I_{T}^{\epsilon}:=I^{\epsilon} \times\right] 0, T\left[. g_{\epsilon}(\mathbf{x})=g(\mathbf{x} / \epsilon)\right.$ is now a periodic wetting boundary condition accounting for the wetting properties of the pore walls. Even under the assumption of periodicity, the microscopic system (10) leads to a high-dimensional problem, since the space discretization parameter needs to be chosen to be much smaller than the characteristic size $\epsilon$ of the heterogeneities of the porous structure, e.g. left-hand side of Figure 1. The homogenization method provides a systematic tool for reducing the intrinsic dimensional complexity by reliably averaging over the microscale represented by a single periodic reference pore $Y$. We note that the nonlinear nature of problem (10) exploits a scale separation with respect to the upscaled chemical potential, see Definition 3.1, for the derivation of the effective macroscopic interfacial evolution in strongly heterogeneous environments. Such a scale separation turns out to be the key for the upscaling/homogenization of nonlinear problems, see [40, 39, 41].

Obviously, the systematic and reliable derivation of practical, convenient, and lowdimensional approximations is the key to feasible numerics of problems posed in porous media and provides a basis for computationally efficient schemes. To this end, we relax the full microscopic formulation (10) further by restricting (10) to periodic fluid flow. By taking the stationary version of equation $(10)_{4}$ on to a single periodic reference pore $Y$ and by denoting the according stationary solution by $\Phi(\cdot)$, we can formulate the following periodic flow problem

$$
\text { (Periodic flow) } \begin{cases}-\mu \Delta_{\mathbf{y}} \mathbf{u}+\nabla_{\mathbf{y}} p=\boldsymbol{\eta} & \text { in } Y^{1}, \\ \operatorname{div}_{\mathbf{y}} \mathbf{u}=0 & \text { in } Y^{1}, \\ \mathbf{u}=\mathbf{0} & \text { on } \partial Y^{2}, \\ \mathbf{u} \text { is } Y^{1} \text {-periodic, } & \\ \operatorname{Pe}\left(\mathbf{u} \cdot \nabla_{\mathbf{y}}\right) \Phi=\lambda \operatorname{div}_{\mathbf{y}}\left(\nabla_{\mathbf{y}}\left(f(\Phi)-\Delta_{\mathbf{y}} \Phi\right)\right) & \text { in } Y^{1}, \\ \nabla_{n} \Phi:=\left(\mathbf{n} \cdot \nabla_{\mathbf{y}}\right) \Phi=g(\mathbf{y}) & \text { on } \partial Y^{2}, \\ \nabla_{n} \Delta_{\mathbf{y}} \Phi=0 & \text { on } \partial Y^{2}, \\ \psi \text { is } Y^{1} \text {-periodic } & \end{cases}
$$

We remark that in certain occasions it might be suitable to further reduce problem 11. For instance, in general the reference cell is only filled by one fluid phase, i.e., 
$\nabla_{\mathbf{y}} \Phi=0$ almost everywhere in $Y^{1}$, and hence one only needs to solve for the periodic Stokes problem $\left.111_{1}-11\right)_{4}$ by replacing the self-induced driving force (8) with the constant driving force $\boldsymbol{\eta}:=\mathbf{e}_{1}$ where $\mathbf{e}_{1}$ denotes the canonical basis vector in the $\mathbf{x}_{1}$ direction of the Euclidean space. The periodic fluid velocity defined by (11) for such an $\eta$ can be considered as the spatially periodic velocity of a moving frame [3]. Motivated by [3, 28, 37], we study the case of large Péclet number and consider the following distinguished case:

Assumption (LP): The Péclet number scales with respect to the characteristic pore size $\epsilon>0$ as follows: $\mathrm{Pe} \sim \frac{1}{\epsilon}$.

Let us first discuss Assumption (LP). If one introduces the microscopic Péclet number $\mathrm{Pe}_{m i c}:=\frac{k \tau \ell \mathrm{U}}{D}$, then it follows immediately that $\mathrm{Pe}=\frac{\mathrm{Pe}_{m i c}}{\epsilon}$. Since we introduced a periodic flow problem on the characteristic length scale $\ell>0$ of the pores by problem (11), it is obvious that we have to apply the microscopic Péclet number in a corresponding microscopic formulation, see 12 below. Hence, the periodic fluid velocity $\mathbf{u}(\mathbf{x} / \epsilon):=\mathbf{u}(\mathbf{y})$ enters the microscopic phase field problem as follows

$$
\text { (Microscopic problem) }\left\{\begin{array}{cc}
\frac{\partial}{\partial t} \phi_{\epsilon}+\frac{\operatorname{Pe}_{\operatorname{mic}}}{\epsilon}(\mathbf{u}(\mathbf{x} / \epsilon) \cdot \nabla) \phi_{\epsilon} & \\
=\lambda \operatorname{div}\left(\nabla\left(f\left(\phi_{\epsilon}\right)-\Delta \phi_{\epsilon}\right)\right) & \text { in } \Omega_{T}^{\epsilon}, \\
\nabla_{n} \phi_{\epsilon}:=\mathbf{n} \cdot \nabla \phi_{\epsilon}=g(\mathbf{x} / \epsilon) & \text { on } I_{T}^{\epsilon}, \\
\nabla_{n} \Delta \phi_{\epsilon}=0 & \text { on } I_{T}^{\epsilon}, \\
\phi_{\epsilon}(\cdot, 0)=\psi(\cdot) & \text { on } \Omega^{\epsilon} .
\end{array}\right.
$$

We note that with our subsequently applied upscaling strategy, we do not account for boundary layers in the vicinity of rigid boundaries. Such boundary layers become increasingly important in the case of large Péclet numbers. Moreover, we make use of the splitting strategy introduced in [42] and here extended to fluid flow, i.e.,

$$
\text { (Splitting) }\left\{\begin{array}{cc}
\frac{\partial}{\partial t}\left(-\Delta_{\epsilon}\right)^{-1} w_{\epsilon}+\frac{\mathrm{Pe}_{\mathrm{mic}}}{\epsilon}(\mathbf{u}(\mathbf{x} / \epsilon) \cdot \nabla)\left(-\Delta_{\epsilon}\right)^{-1} w_{\epsilon} & \\
=\lambda\left(\operatorname{div}\left(\hat{\mathrm{M}} \nabla w_{\epsilon}\right)+\operatorname{div}\left(\hat{\mathrm{M}} \nabla f\left(\phi_{\epsilon}\right)\right)\right) & \text { in } \Omega_{T}^{\epsilon}, \\
-\nabla_{n} \Delta \phi_{\epsilon}=\nabla_{n} w_{\epsilon}=0 & \text { on } I_{T}^{\epsilon}, \\
-\Delta_{\epsilon} \phi_{\epsilon}=w_{\epsilon} & \\
\nabla_{n} \phi_{\epsilon}=g(\mathbf{x} / \epsilon)=g_{\epsilon}(\mathbf{x}) & \text { on } I_{T}^{\epsilon}, \\
\phi_{\epsilon}(\cdot, 0)=\psi(\cdot) & \text { on } \Omega^{\epsilon},
\end{array}\right.
$$

where we will properly define $\Delta_{\epsilon}=\mathcal{A}_{\epsilon}$ in Section 4 .

The main result of our study is the systematic derivation of upscaled immiscible flow equations which effectively account for the pore geometry starting from the microscopic system (11)-(12), i.e.,

$$
\text { (Upscaled equation) }\left\{\begin{array}{r}
p \frac{\partial \phi_{0}}{\partial t}-\operatorname{div}\left(\hat{\mathrm{C}} \nabla \phi_{0}\right)=\lambda \operatorname{div}\left(\hat{\mathrm{M}}_{\phi} \nabla f\left(\phi_{0}\right)\right) \\
-\frac{\lambda}{p} \operatorname{div}\left(\hat{\mathrm{M}}_{w} \nabla\left(\operatorname{div}\left(\hat{\mathrm{D}} \nabla \phi_{0}\right)-\tilde{g}_{0}\right)\right)
\end{array}\right.
$$


where $\hat{\mathrm{C}}$ takes the fluid convection into account. These two tensors account for the socalled diffusion-dispersion relations (e.g. Taylor-Aris-dispersion [4, 10, 48]). The result (14) makes use of the recently proposed splitting strategy for the homogenization of fourth order problems in [42] and an asymptotic multiscale expansion with drift (i.e., moving frame) introduced in [3, 27].

The manuscript is organized as follows. We present our main results in Section 3 and the corresponding proofs follow in the subsequent Section 4 . Concluding remarks and open questions are offered in Section 6.

\section{Preliminaries and notation}

We recall basic results required for our subsequent analysis which depends also on certain notational conventions. We consider connected macroscopic domains $\Omega$ with Lipschitz continuous boundaries $\partial \Omega$. Under the usual conventions for Sobolev spaces, we say that $u \in W^{k, p}(\Omega)$ if and only if

$$
\|u\|_{k, p}^{p}:=\sum_{|\alpha| \leq k}\left\|D^{\alpha} u\right\|_{L^{p}}^{p}<\infty
$$

for a multi-index $\alpha$ such that $D^{\alpha}:=\frac{\partial^{|\alpha|}}{\partial^{\alpha} x_{1} \ldots \partial^{\alpha} d x_{d}}$ and $p<\infty$. Herewith, we can identify corresponding Hilbert spaces $(p=2)$ by $H^{k}(\Omega):=W^{k, 2}(\Omega)$. We introduce the following (energy) space of functions

$$
H_{E}^{2}(\Omega):=\left\{v \in H^{2}(\Omega) \mid \nabla_{n} v=0 \quad \text { on } \quad \partial \Omega\right\}
$$

which naturally appears in the context of weak solutions for the phase field equations (7) 4 . In order to account for the periodic reference cells appearing due to asymptotic multiscale-expansions/homogenization, we define $\bar{H}_{p e r}^{1}(Y)$ as the closure of $C_{\text {per }}^{\infty}(Y)$ in the $H^{1}$-norm where $C_{p e r}^{\infty}(Y)$ is the subset of $Y$-periodic functions of $C^{\infty}\left(\mathbb{R}^{d}\right)$. As we need uniqueness of solutions, we will work with the following space of functions

$$
H_{p e r}^{1}(Y):=\left\{u \in \bar{H}_{p e r}^{1}(Y) \mid \mathcal{M}_{Y}(u)=0\right\}
$$

where $\mathcal{M}_{Y}(u):=\frac{1}{|Y|} \int_{Y} u d \mathbf{y}$. In order to establish the existence and uniqueness of weak solutions of the upscaled convective phase field equations, we need the following Aubin-Lions compactness result (e.g. [44]), i.e.,

Theorem 2.1 (Aubin-Lions) Let $X_{0}, X, X_{1}$ be Banach spaces with $X_{0} \subset X \subset$ $X_{1}$ and assume that $X_{0} \hookrightarrow X$ is compact and $X \hookrightarrow X_{1}$ is continuous. Let $1<p<\infty, 1<q<\infty$ and let $X_{0}$ and $X_{1}$ be reflexive. Then, for $W:=$ $\left\{u \in L^{p}\left(0, T ; X_{0}\right) \mid \partial_{t} u \in L^{q}\left(0, T ; X_{1}\right)\right\}$ the inclusion $W \hookrightarrow L^{p}(0, T ; X)$ is compact. 
Upscaling phase field equations for periodic fluid flow

\section{Results: Effective immiscible flow equations in porous media}

The presentation of our main result depends on the following:

Definition 3.1 (Scale separation) We say that the the macroscopic chemical potential is scale separated if and only if the upscaled chemical potential

$$
\mu_{0}:=f(\phi)-\Delta \phi
$$

satisfies $\frac{\partial \mu_{0}}{\partial x_{l}}=0$ for each $1 \leq l \leq d$ on the level of the reference cell $Y$ but not in the macroscopic domain $\Omega$.

Remark 3.2 We note that the scale separation in Definition 3.1 follows intuitively from the key requirement in homogenization theory that one can identify a slow (macroscopic) variable and at least one fast (microscopic) variable. Hence, the above scale separation means that the macroscopic variable does not vary over the dimension of the microscale defined by a characteristic reference cell.

The scale separation (3.1) emerges as a key requirement for the homogenization of nonlinearly coupled partial differential equations in order to guarantee the mathematical well-posedness of the corresponding cell problems which define effective transport coefficients in homogenized, nonlinear (and coupled) problems [40, 39, 41].

We note that the upscaling requires to identify effective macroscopic boundary conditions on the macroscopic domain $\Omega$. Such a condition will be denoted by $\tilde{h}_{0}$ below. In fact, $\tilde{h}_{0}$ can be computed as $\tilde{g}_{0}$. We summarize our main result in the following

Theorem 3.3 (Effective convective Cahn-Hilliard equation) We assume that the Assumption (LP) holds and that the macroscopic chemical potential $\mu_{0}$ satisfies the scale separation property in the sense of Definition 3.1 and let $\psi(\mathbf{x}) \in H^{2}(\Omega)$.

Then, the microscopic equations (11)-(12) for immiscible flow in porous media admit the following effective macroscopic form after averaging over the microscale, i.e.,

$$
\begin{cases}p \frac{\partial \phi_{0}}{\partial t}-\operatorname{div}\left(\hat{\mathrm{C}} \nabla \phi_{0}\right)=\lambda \operatorname{div}\left(\hat{\mathrm{M}}_{\phi} \nabla f\left(\phi_{0}\right)\right) & \\ -\frac{\lambda}{p} \operatorname{div}\left(\hat{\mathrm{M}}_{w} \nabla\left(\operatorname{div}\left(\hat{\mathrm{D}} \nabla \phi_{0}\right)-\tilde{g}_{0}\right)\right) & \text { in } \Omega_{T}, \\ \nabla_{n} \phi_{0}:=\mathbf{n} \cdot \nabla \phi_{0}=\tilde{h}_{0}(\mathbf{x}) & \text { on } \partial \Omega \times] 0, T[, \\ \nabla_{n} \Delta \phi_{0}=0 & \text { on } \partial \Omega \times] 0, T[, \\ \phi_{0}(\cdot, 0)=\psi(\cdot) & \text { in } \Omega,\end{cases}
$$

where the tensors $\hat{\mathrm{C}}:=\left\{\mathrm{c}_{i k}\right\}_{1 \leq i, k \leq d}, \hat{\mathrm{D}}:=\left\{\mathrm{d}_{i k}\right\}_{1 \leq i, k \leq d}, \hat{\mathrm{M}}_{\phi}=\left\{\mathrm{m}_{i k}^{\phi}\right\}_{1 \leq i, k \leq d}$, and 
$\hat{\mathrm{M}}_{w}=\left\{\mathrm{m}_{i k}^{w}\right\}_{1 \leq i, k \leq d}$ are defined by

$$
\left\{\begin{aligned}
\mathrm{c}_{i k} & :=\frac{\mathrm{Pe}_{m i c}}{|Y|} \int_{Y^{1}}\left(\mathrm{u}^{i}-\mathrm{v}^{i}\right) \delta_{i k} \xi_{\phi}^{k} d \mathbf{y} \\
\mathrm{d}_{i k} & :=\frac{1}{|Y|} \sum_{j=1}^{d} \int_{Y^{1}}\left(\delta_{i k}-\delta_{i j} \frac{\partial \xi_{\phi}^{k}}{\partial y_{j}}\right) d \mathbf{y} \\
\mathrm{m}_{i k}^{\phi} & :=\frac{1}{|Y|} \sum_{j=1}^{d} \int_{Y^{1}}\left(\mathrm{~m}_{i k}-\mathrm{m}_{i j} \frac{\partial \xi_{\phi}^{k}}{\partial y_{j}}\right) d \mathbf{y} \\
\mathrm{m}_{i k}^{w} & :=\frac{1}{|Y|} \sum_{j=1}^{d} \int_{Y^{1}}\left(\mathrm{~m}_{i k}-\mathrm{m}_{i j} \frac{\partial \xi_{w}^{k}}{\partial y_{j}}\right) d \mathbf{y}
\end{aligned}\right.
$$

The effective fluid velocity $\mathbf{v}$ is defined by $\mathrm{v}^{j}:=\frac{\mathrm{Pe}_{\operatorname{mic}}}{\left|Y^{1}\right|} \int_{Y^{1}} \mathrm{u}^{j}(\mathbf{y}) d \mathbf{y}$ where $\mathbf{u}$ solves the periodic reference cell problem (11). The effective wetting boundary condition on the pore walls becomes $\tilde{g}_{0}:=-\frac{\gamma}{C_{h}} \frac{1}{|Y|} \int_{\partial Y^{1}}\left(a_{1} \chi_{\partial Y_{w}^{1}}(\mathbf{y})+a_{2} \chi_{\partial Y_{w}^{2}}(\mathbf{y})\right) d \mathbf{y}$ and on the boundary $\partial \Omega$ of the macroscopic domain $\Omega$ we impose $\tilde{h}_{0}:=-\frac{\gamma}{C_{h}} \frac{1}{|Y|} \int_{\Gamma}\left(a_{\Gamma}(\mathbf{y})\right) d \mathbf{y}$. The corrector functions $\xi_{\phi}^{k} \in H_{p e r}^{1}\left(Y^{1}\right)$ and $\xi_{w}^{k} \in L^{2}\left(\Omega ; H_{p e r}^{1}\left(Y^{1}\right)\right)$ for $1 \leq k, l \leq d$ solve in the distributional sense the following reference cell problems

$$
\begin{aligned}
& \xi_{\phi}^{k}: \begin{cases}-\sum_{i, j=1}^{d} \frac{\partial}{\partial y_{i}}\left(\delta_{i k}-\delta_{i j} \frac{\partial \xi_{\phi}^{k}}{\partial y_{j}}\right)=0 & \text { in } Y^{1}, \\
\sum_{i, j=1}^{d} \mathrm{n}_{i}\left(\delta_{i j} \frac{\partial \xi_{\phi}^{k}}{\partial y_{j}}-\delta_{i k}\right)=0 & \text { on } \partial Y^{1}, \\
\xi_{\phi}^{k}(\mathbf{y}) \text { is } Y \text {-periodic and } \mathcal{M}_{Y^{1}}\left(\xi_{\phi}^{k}\right)=0, & \end{cases} \\
& \xi_{w}^{k}:\left\{\begin{array}{c}
-\sum_{i, j=1}^{d} \frac{\partial}{\partial y_{i}}\left(\delta_{i k}-\delta_{i j} \frac{\partial \xi_{w}^{k}}{\partial y_{j}}\right) \\
=\lambda\left(\sum_{i, j=1}^{d} \frac{\partial}{\partial y_{i}}\left(\mathrm{~m}_{i k}-\mathrm{m}_{i j} \frac{\partial \xi_{\phi}^{k}}{\partial y_{j}}\right) \quad \text { in } Y^{1},\right. \\
\sum_{i, j=1}^{d} \mathrm{n}_{i}\left(\left(\delta_{i j} \frac{\partial \xi_{w}^{k}}{\partial y_{j}}-\delta_{i k}\right)\right. \\
-\lambda \sum_{i, j=1}^{d} \frac{\partial}{\partial y_{i}}\left(\mathrm{~m}_{i k}-\mathrm{m}_{i j} \frac{\partial \xi_{\phi}^{k}}{\partial y_{j}}\right)=0 \quad \text { on } \partial Y^{1}, \\
\xi_{w}^{k}(\mathbf{y}) \text { is } Y \text {-periodic and } \mathcal{M}_{Y^{1}}\left(\xi_{w}^{k}\right)=0 .
\end{array}\right.
\end{aligned}
$$

Remark 3.4 (Isotropic mobility) The cell problem (22) is equal to problem (21) if we consider the case of isotropic mobility tensors, i.e., $\hat{\mathrm{M}}:=m \hat{\mathrm{I}}$. In this special case, we immediately have $\xi_{\phi}^{k}=\xi_{w}^{k}$ and hence the porous media correction tensors satisfy $m \hat{\mathrm{D}}=\hat{\mathrm{M}}_{\phi}=\hat{\mathrm{M}}_{w}$.

The next theorem guarantees the well-posedness of 23 in the sense of week solutions. For convenience, we achieve existence of weak solutions for polynomial free energies in the sense of Assumption (PF) [49].

Theorem 3.5 (Existence \& Uniqueness) Let $\psi \in L^{2}(\Omega), T^{*}>0$, and assume that the admissible free energy densities $F$ in (1) satisfy Assumption (PF). Then, there exists a unique solution $\phi_{0} \in L^{\infty}(] 0, T^{*}\left[; L^{2}(\Omega)\right) \cap L^{2}\left(\left[0, T^{*}\left[; H_{E}^{2}(\Omega)\right)\right.\right.$ to the following 
upscaled/homogenized problem

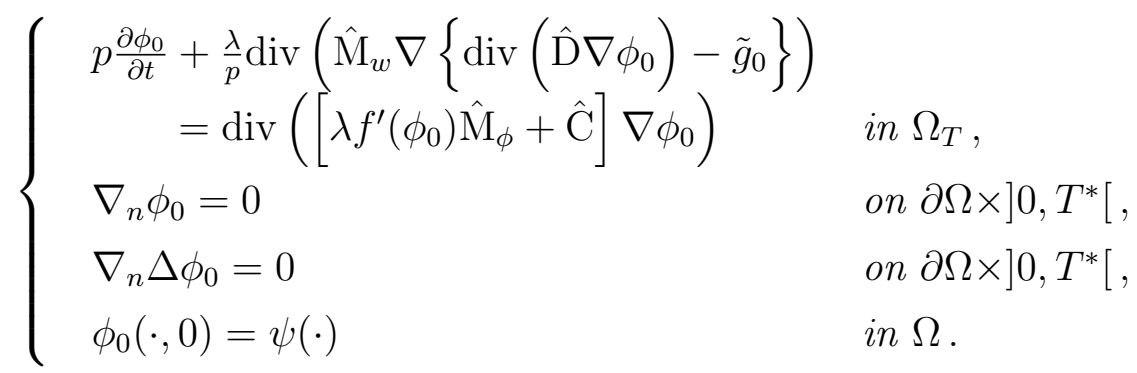

We prove Theorem 3.5 by adapting arguments from [49, Section 2, p.151] to the homogenized setting including the diffusion-dispersion tensor which accounts for periodic flow.

\subsection{Numerical computations}

To exemplify the results presented above we perform a numerical study of the effective macroscopic Cahn-Hilliard equation (19). We consider a two-dimensional (2D) porous medium consisting of a series of periodic reference cells the geometrical shape of which is a non-straight channel of constant cross-section (see Figure 2) with periodic boundary conditions at the inlet and outlet. The Cartesian coordinates in the microscopic problem are named as $x$ and $y$ which correspond to the $y_{1}$ and $y_{2}$ variables used in the definition of the domain $Y^{1}$, respectively. We define the geometry in such a way that the porosity of the medium is $p=0.46$. The macroscopic domain $\Omega$ is compound of 35 reference cells in the perpendicular direction $y$ of the flow and 50 in the $x$ direction. We fix a constant driving force $U=1$ at the inlet of the system by fixing the gradient of the chemical potential[51]. For simplicity we take the macroscopic mobility $M=1$ which gives rise to a microscopic Péclet number $\mathrm{Pe}_{m i c}=0.04$.
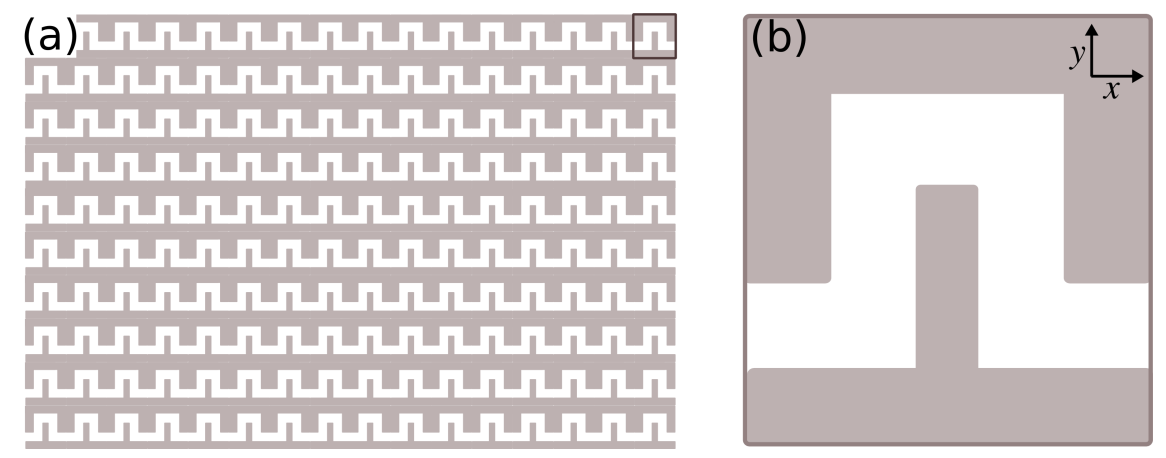

Figure 2. (a): Example of the 2D porous medium considered for the numerical computations. The reference cell consists of a non-straight channel of constant crosssection which is periodic in the $x$ direction as depicted in (b). Gray area corresponds to the solid phase of the medium.

We first compute the components of the different tensors $\hat{\mathrm{C}}, \hat{\mathrm{D}}, \hat{\mathrm{M}}_{\phi}$, and $\hat{\mathrm{M}}_{w}$ for which we need to solve the reference cell problems (21) and (22). We consider the case 
of isotropic mobility with $m=1$ and hence we have $\xi_{\phi}^{k}=\xi_{w}^{k}$ and $\hat{D}=\hat{M}_{\phi}=\hat{M}_{w}$. In this case, the reference cell problem is reduced to:

$$
\xi_{\phi}^{k}: \begin{cases}\left(\frac{\partial^{2}}{\partial x^{2}}+\frac{\partial^{2}}{\partial y^{2}}\right) \xi_{\phi}^{k}=0 & \text { in } Y^{1}, \\ \left(\mathrm{n}_{1} \frac{\partial}{\partial x}+\mathrm{n}_{2} \frac{\partial}{\partial y}\right) \xi_{\phi}^{k}=\mathrm{n}_{k} & \text { on } \partial Y^{1}\end{cases}
$$

which corresponds to the Laplace equation with special boundary conditions. The above equation is solved by using a finite differences numerical scheme and the resulting corrector functions $\xi_{\phi}^{1}(x, y)$ and $\xi_{\phi}^{2}(x, y)$ are plotted in Figure 3 .
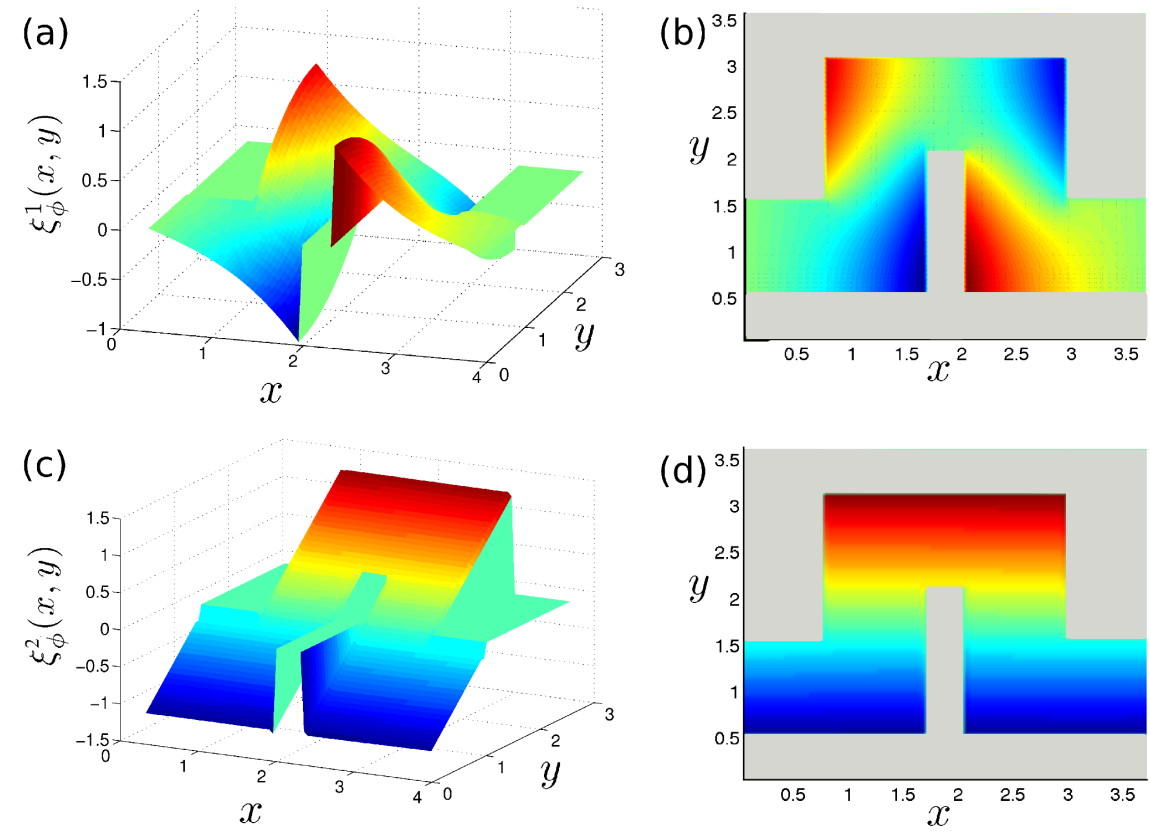

Figure 3. Corrector functions $\xi_{\phi}^{1}(x, y)(\mathrm{a}, \mathrm{b})$ and $\xi_{\phi}^{2}(x, y)(\mathrm{c}, \mathrm{d})$ for the particular reference cell defined in Figure 2b. Panels (a) and (c) show the three-dimensional plots, and panels (b) and (d) show the corresponding $2 \mathrm{D}$ projection onto the plane $(x, y)$.

Once we know the corrector functions of the reference cell problem, we can compute the different elements of the tensor $\hat{D}$ as defined in 20 obtaining the values $\mathrm{d}_{11}=0.4$, and $\mathrm{d}_{12}=\mathrm{d}_{21}=\mathrm{d}_{22}=0$. Note that these are similar values to those reported in [6] for a similar porous geometry. Next we solve the Stokes flow for this particular microscopic geometry by numerically integrating the periodic reference cell problem (11) to obtain the velocity $\mathbf{u}$ and hence the coefficients for the tensor $\hat{\mathrm{C}}$. The results for the two velocity components $\mathrm{u}^{1}(x, y)$ and $\mathrm{u}^{2}(x, y)$ are presented in Figure 4. By applying the formula given in 20 we obtain the coefficients $c_{11}=0.015$ and $c_{22}=0.023$. Note that by definition $\mathrm{c}_{12}=\mathrm{c}_{21}=0$.

Finally, with all the different tensor coefficients we can numerically integrate the problem (19) in the macroscopic domain $\Omega$, the Cartesian coordinates of which are denoted as $(X, Y)$. We use a finite difference scheme for the spatial discretization and 

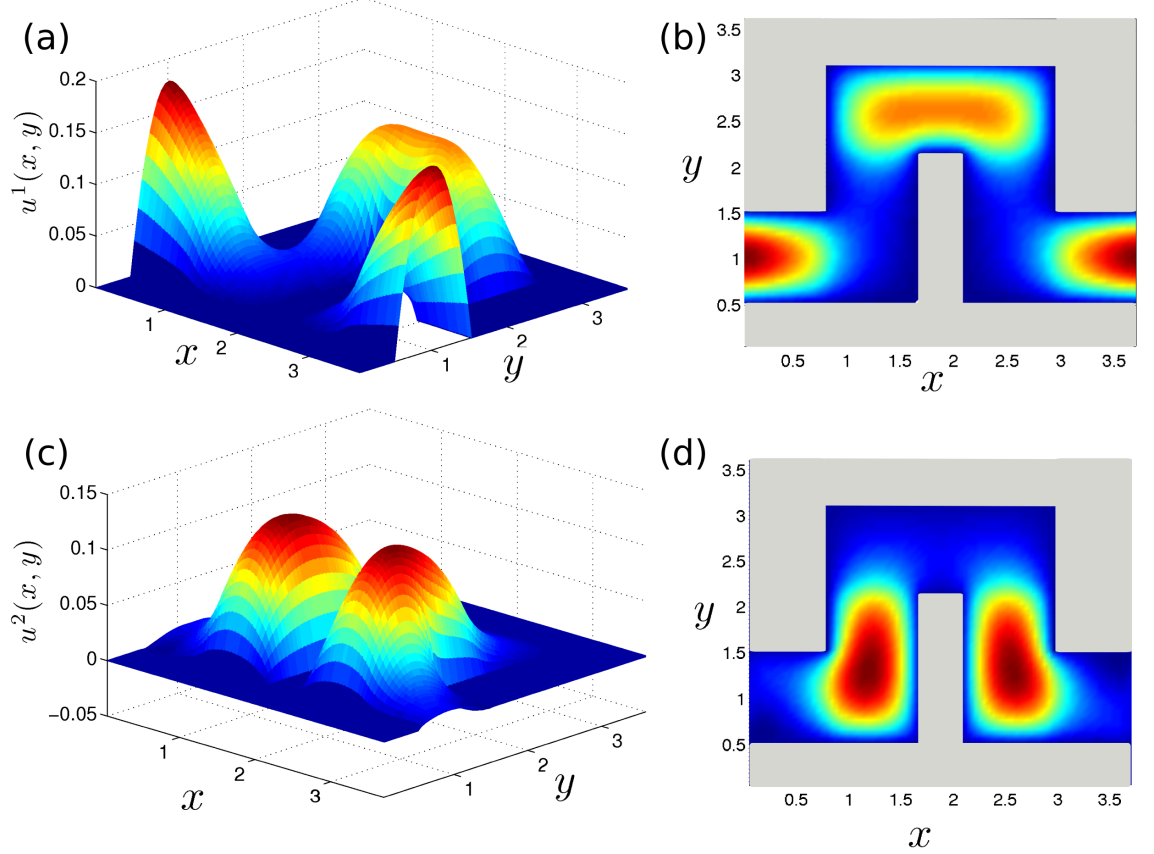

Figure 4. Two components of the velocity field $\mathrm{u}^{1}(x, y)(\mathrm{a}, \mathrm{b})$ and $\mathrm{u}^{2}(x, y)(\mathrm{c}, \mathrm{d})$. Panels (a) and (c) show the three-dimensional plots, and panels (b) and (d) show the corresponding $2 \mathrm{D}$ projection onto the plane $(x, y)$.

a fourth-order Runge-Kutta algorithm with adaptive time stepsize to march froward in time. The domain is discretized with a grid spacing $\Delta X=0.01$ and we impose periodic boundary conditions along the transversal direction of the flow. As an initial condition, we consider a small sinusoidal shape for the interface separating the liquid from the gas phase. Also, to simulate the same condition as in the porous medium, we impose the driving force $U$ to be fixed alternately at the inlet of the system in such a way it follows the periodicity of microscopic structure. The evolution of the interface position is then found by setting $\phi_{0}(X, Y, t)=0$. The results are presented in Figure 5 where we observe that the profile of the interface evolves into a well defined spatial periodic shape which corresponds to the periodic porous medium that is defined at the microscopic level (had the macroscopic model ignored the microscopic details, by e.g. taking the tensors to be identity matrices, the interface would be flat at all times). For large times and after the influence of the initial disturbances dies out, the interface approaches a steady travelling front with a microstructure that reflects the porous medium structure (as expected). Our results hence show that the effective macroscopic equation is able to retain the microscopic details even though we do not resolve them numerically. 


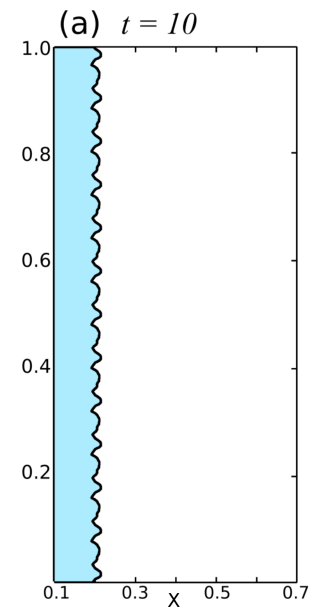

(b) $t=50$

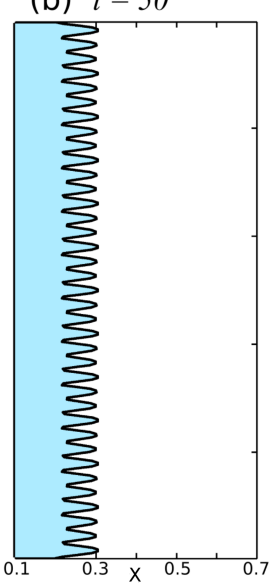

(c) $t=100$

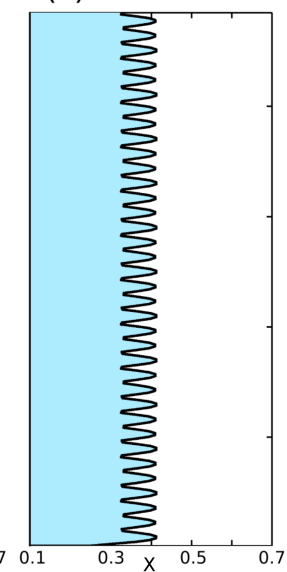

(d) $t=150$

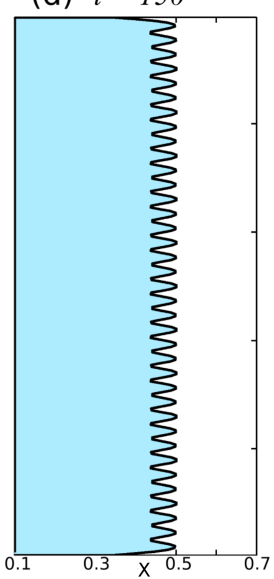

Figure 5. Numerical integrations of the effective macroscopic Cahn-Hilliard equation at different times. Blue color represents the liquid phase and the interface position is depicted as a solid black line.

\section{Formal derivation of Theorem 3.3}

As in [42], we introduce the differential operators

$$
\begin{array}{rlrl}
\mathcal{A}_{0}= & -\sum_{i, j=1}^{d} \frac{\partial}{\partial y_{i}}\left(\delta_{i j} \frac{\partial}{\partial y_{j}}\right), & \mathcal{B}_{0}=-\sum_{i, j=1}^{d} \frac{\partial}{\partial y_{i}}\left(\mathrm{~m}_{i j} \frac{\partial}{\partial y_{j}}\right), \\
\mathcal{A}_{1}=-\sum_{i, j=1}^{d}\left[\frac{\partial}{\partial x_{i}}\left(\delta_{i j} \frac{\partial}{\partial y_{j}}\right)\right. & \mathcal{B}_{1}=-\sum_{i, j=1}^{d}\left[\frac{\partial}{\partial x_{i}}\left(\mathrm{~m}_{i j} \frac{\partial}{\partial y_{j}}\right)\right. \\
\left.+\frac{\partial}{\partial y_{i}}\left(\delta_{i j} \frac{\partial}{\partial x_{j}}\right)\right], & & \left.+\frac{\partial}{\partial y_{i}}\left(\mathrm{~m}_{i j} \frac{\partial}{\partial x_{j}}\right)\right], \\
\mathcal{A}_{2}=-\sum_{i, j=1}^{d} \frac{\partial}{\partial x_{j}}\left(\delta_{i j} \frac{\partial}{\partial x_{j}}\right), & \mathcal{B}_{2}=-\sum_{i, j=1}^{d} \frac{\partial}{\partial x_{j}}\left(\mathrm{~m}_{i j} \frac{\partial}{\partial x_{j}}\right),
\end{array}
$$

which make use of the micro-scale $\frac{\mathbf{x}}{\epsilon}=: \mathbf{y} \in Y$ such that $\mathcal{A}_{\epsilon}:=\epsilon^{-2} \mathcal{A}_{0}+\epsilon^{-1} \mathcal{A}_{1}+\mathcal{A}_{2}$, and $\mathcal{B}_{\epsilon}:=\epsilon^{-2} \mathcal{B}_{0}+\epsilon^{-1} \mathcal{B}_{1}+\mathcal{B}_{2}$. Herewith, the Laplace operators $\Delta$ and $\operatorname{div}(\hat{\mathrm{M}} \nabla)$ become $\Delta u^{\epsilon}(\mathbf{x})=\mathcal{A}_{\epsilon} u(\mathbf{x}, \mathbf{y})$ and $\operatorname{div}(\hat{\mathrm{M}} \nabla) u^{\epsilon}(\mathbf{x})=\mathcal{B}_{\epsilon} u(\mathbf{x}, \mathbf{y})$, respectively, where $u^{\epsilon}(\mathbf{x}, t):=u\left(\mathbf{x}-\frac{\mathbf{v}}{\epsilon} t, \mathbf{y}, t\right)$. Due to the drift [3, 27], we additionally have

$$
\frac{\partial}{\partial t} u^{\epsilon}=\left(\frac{\partial}{\partial t}-\frac{\mathbf{v} \cdot \nabla_{\mathbf{x}}}{\epsilon}\right) u^{\epsilon}
$$

where we find below by the Fredholm alternative (or a solvability constraint) that $\mathbf{v}:=\frac{P_{\text {loc }}}{\left|Y^{1}\right|} \int_{Y^{1}} \mathrm{u}^{j}(y) d y$. As in [42] we apply the method of formal asymptotic multiscale expansions, that is,

$$
\begin{aligned}
w^{\epsilon} & :=w_{0}(\mathbf{x}, \mathbf{y}, t)+\epsilon w_{1}(\mathbf{x}, \mathbf{y}, t)+\epsilon^{2} w_{2}(\mathbf{x}, \mathbf{y}, t)+\ldots, \\
\phi^{\epsilon} & :=\phi_{0}(\mathbf{x}, \mathbf{y}, t)+\epsilon \phi_{1}(\mathbf{x}, \mathbf{y}, t)+\epsilon^{2} \phi_{2}(\mathbf{x}, \mathbf{y}, t)+\ldots,
\end{aligned}
$$

together with the splitting strategy introduced therein. In order to cope with the nonlinear form of the homogeneous free energy $f=F^{\prime}$, see (2) and (3), we make use of 
a Taylor expansion which naturally leads to an expansion in $\epsilon$, i.e.,

$$
f\left(\phi^{\epsilon}\right)=f\left(\phi_{0}\right)+f^{\prime}\left(\phi_{0}\right)\left(\phi^{\epsilon}-\phi_{0}\right)+\frac{1}{2} f^{\prime \prime}\left(\phi_{0}\right)\left(\phi^{\epsilon}-\phi_{0}\right)^{2}+\mathcal{O}\left(\left(\phi^{\epsilon}-\phi_{0}\right)^{3}\right) .
$$

As a consequence, we obtain the following sequence of problems by comparing terms of the same order in $\epsilon$, with the first three problems being,

$$
\begin{aligned}
& \mathcal{O}\left(\epsilon^{-2}\right): \begin{cases}\left.\lambda \mathcal{B}_{0}\left[w_{0}+f\left(\phi_{0}\right)\right]+\mathrm{Pe}_{\text {mic }}(\mathbf{u} \cdot \nabla)_{\mathbf{y}}\right) \mathcal{A}_{2}^{-1} w_{0}=0 & \text { in } Y^{1}, \\
& \text { no flux b.c. }, \\
& w_{0} \text { is } Y^{1} \text {-periodic, } \\
\mathcal{A}_{0} \phi_{0}=0 & \text { in } Y^{1}, \\
\nabla_{n} \phi_{0}=0 & \text { on } \partial Y_{w}^{1} \cap \partial Y_{w}^{2}, \\
\phi_{0} \text { is } Y^{1} \text {-periodic, } & \end{cases} \\
& \mathcal{O}\left(\epsilon^{-1}\right): \begin{cases}\lambda \mathcal{B}_{0}\left[w_{1}+f^{\prime}\left(\phi_{0}\right) \phi_{1}\right]+\operatorname{Pe}_{\text {mic }}\left(\mathbf{u} \cdot \nabla_{\mathbf{y}}\right) \mathcal{A}_{2}^{-1} w_{1} & \\
=-\lambda \mathcal{B}_{1}\left[w_{0}+f\left(\phi_{0}\right)\right] & \\
\quad-\operatorname{Pe}_{\text {mic }}((\mathbf{u}-\mathbf{v}) \cdot \nabla) \mathcal{A}_{2}^{-1} w_{0} & \text { in } Y^{1}, \\
\text { no flux b.c. } & \\
w_{1} \text { is } Y^{1} \text {-periodic, } & \text { in } Y^{1} \\
\mathcal{A}_{0} \phi_{1}=-\mathcal{A}_{1} \phi_{0} & \text { on } \partial Y_{w}^{1} \cap \partial Y_{w}^{2}, \\
\nabla_{n} \phi_{1}=0 & \\
\phi_{1} \text { is } Y^{1} \text {-periodic }, & \end{cases}
\end{aligned}
$$

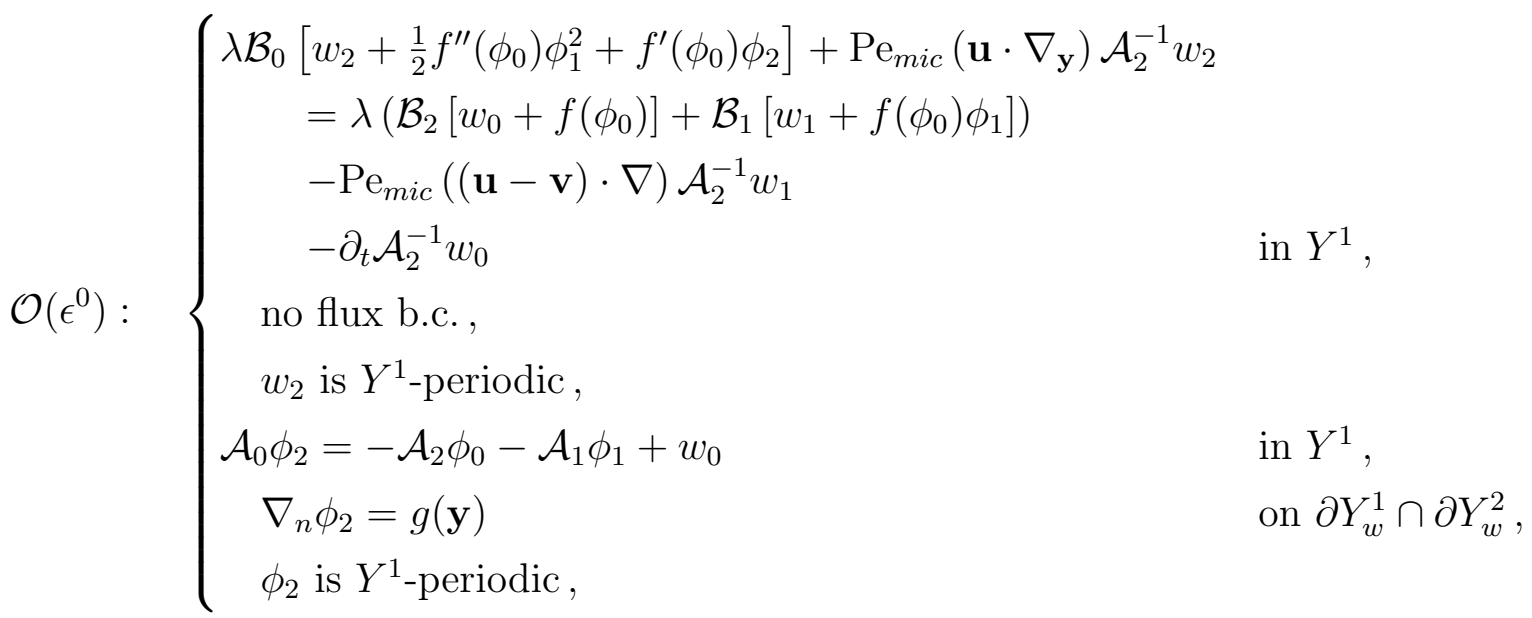

As usual, the first problem (29) induces that the leading order terms $\phi_{0}$ and $w_{0}$ are independent of the micro-scale $\mathbf{y}$. The second problem 30 reads in explicit form for 
$\phi_{1}$ as follows,

$$
\xi_{\phi}:\left\{\begin{array}{cl}
-\sum_{i, j=1}^{d} \frac{\partial}{\partial y_{i}}\left(\delta_{i k}-\delta_{i j} \frac{\partial \xi_{\phi}^{k}}{\partial y_{j}}\right)= & \\
=-\operatorname{div}\left(\mathbf{e}_{k}-\nabla_{y} \xi_{\phi}^{k}\right)=0 & \text { in } Y^{1} \\
\mathbf{n} \cdot\left(\nabla \xi_{\phi}^{k}+\mathbf{e}_{k}\right)=0 & \text { on } \partial Y_{w}^{1} \cap \partial Y_{w}^{2} \\
\xi_{\phi}^{k}(\mathbf{y}) \text { is } Y \text {-periodic and } \mathcal{M}_{Y^{1}}\left(\xi_{\phi}^{k}\right)=0, &
\end{array}\right.
$$

which represents the reference cell problem for $\phi_{0}$ after identifying $\phi_{1}=$ $-\sum_{k=1}^{d} \xi_{\phi}^{k}(\mathbf{y}) \frac{\partial \phi_{0}}{\partial x_{k}}$.

The cell problem for $w_{1}$ is substantially more involved since it depends on the fluid velocity $\mathbf{u}$ and the the corrector $\xi_{\phi}^{k}$ from 32 . Specifically,

$$
\begin{aligned}
-\sum_{k, i, j=1}^{d} \frac{\partial}{\partial y_{i}}\left(\mathrm{~m}_{i j}\left(\frac{\partial x_{k}}{\partial x_{j}}-\frac{\partial \xi_{w}^{k}}{\partial y_{j}}\right) \frac{\partial w_{0}}{\partial x_{k}}\right)= & \sum_{k, i, j=1}^{d} \frac{\partial}{\partial y_{i}}\left(\mathrm{~m}_{i j}\left(\frac{\partial x_{k}}{\partial x_{j}}-\frac{\partial \xi_{\phi}^{k}}{\partial y_{j}}\right) \frac{\partial f\left(\phi_{0}\right)}{\partial x_{k}}\right) \\
& -\operatorname{Pe}_{m i c} \sum_{i=1}^{d}\left(\mathrm{u}^{i}-\mathrm{v}^{i}\right) \frac{\partial \phi_{0}}{\partial x_{i}} \quad \text { in } Y^{1},
\end{aligned}
$$

which can be simplified under a scale seperated chemical potential in the sense of Definition 3.1. i.e., $\frac{\partial}{\partial x_{k}} f(\phi)=f^{\prime}(\phi) \frac{\partial \phi}{\partial x_{k}}=\frac{\partial w}{\partial x_{k}} \quad$ for $1 \leq k \leq d$, to the following cell problem,

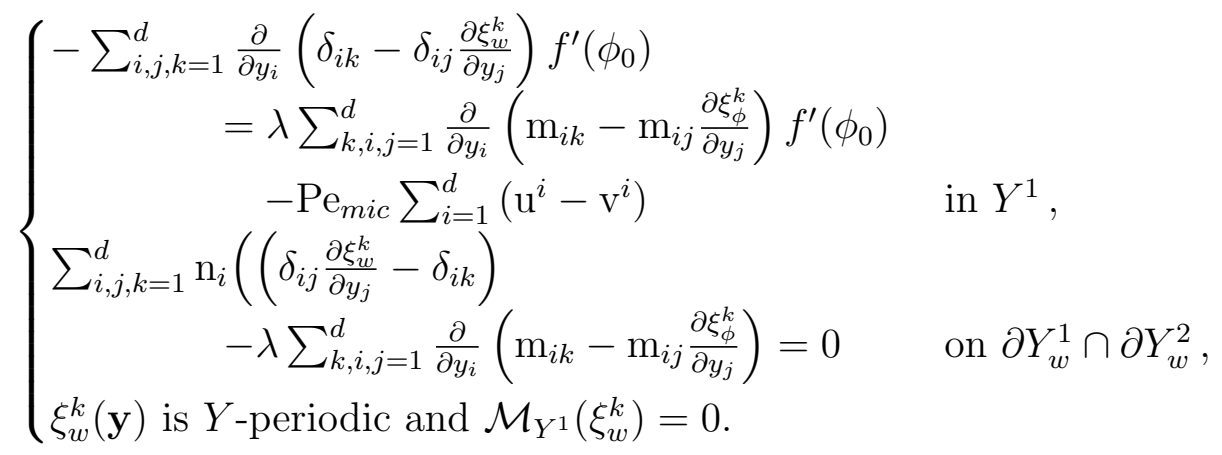

A solvability constraint (e.g. the Fredholm alternative) immediately turns (34) into the following characterization of $\xi_{w}^{k}$ and $\mathrm{v}^{i}$, i.e.,

$$
\begin{aligned}
& \mathrm{v}^{j}:=\frac{\mathrm{Pe}_{\mathrm{mic}}}{\left|Y^{1}\right|} \int_{Y^{1}} \mathrm{u}^{j}(\mathbf{y}) d \mathbf{y} \\
& \left\{\begin{array}{cl}
-\sum_{i, j, k=1}^{d} \frac{\partial}{\partial y_{i}}\left(\delta_{i k}-\delta_{i j} \frac{\partial \xi_{w}^{k}}{\partial y_{j}}\right)=\lambda \sum_{k, i, j=1}^{d} \frac{\partial}{\partial y_{i}}\left(\mathrm{~m}_{i k}-\mathrm{m}_{i j} \frac{\partial \xi_{\phi}^{k}}{\partial y_{j}}\right) & \text { in } Y^{1} \\
\sum_{i, j, k=1}^{d} \mathrm{n}_{i}\left(\left(\delta_{i j} \frac{\partial \xi_{w}^{k}}{\partial y_{j}}-\delta_{i k}\right)-\lambda \sum_{k, i, j=1}^{d} \frac{\partial}{\partial y_{i}}\left(\mathrm{~m}_{i k}-\mathrm{m}_{i j} \frac{\partial \xi_{\phi}^{k}}{\partial y_{j}}\right)=0\right. & \text { on } \partial Y_{w}^{1} \cap \partial Y_{w}^{2}, \\
\xi_{w}^{k}(\mathbf{y}) \text { is } Y \text {-periodic and } \mathcal{M}_{Y^{1}}\left(\xi_{w}^{k}\right)=0 . &
\end{array}\right.
\end{aligned}
$$


We are then left to study the last problem (31) arising by the asymptotic multiscale expansions. Problem $(31)_{2}$ for $\phi_{2}$ is classical and leads immediately to the upscaled equation

$$
-\Delta_{\hat{\mathrm{D}}} \phi_{0}:=-\operatorname{div}\left(\hat{\mathrm{D}} \nabla \phi_{0}\right)=p w_{0}+\tilde{g}_{0}
$$

see also [42, where the porous media correction tensor $\hat{\mathrm{D}}:=\left\{\mathrm{d}_{i k}\right\}_{1 \leq i, k \leq d}$ is defined by

$$
|Y| \mathrm{d}_{i k}:=\sum_{j=1}^{d} \int_{Y^{1}}\left(\delta_{i k}-\delta_{i j} \frac{\partial \xi_{\phi}^{k}}{\partial y_{j}}\right) d \mathbf{y} .
$$

Next, we apply the Fredholm alternative on equation 31 , i.e.,

$$
\begin{aligned}
& \int_{Y^{1}}\left\{\lambda\left(\mathcal{B}_{2} w_{0}+\mathcal{B}_{1} w_{1}\right)-\lambda \mathcal{B}_{1}\left[f\left(\phi_{0}\right) \phi_{1}\right]-\lambda \mathcal{B}_{2} f\left(\phi_{0}\right)-\partial_{t} \mathcal{A}_{2}^{-1} w_{0}\right. \\
& \left.-\mathrm{Pe}_{m i c}\left(\mathbf{u} \cdot \nabla_{\mathbf{y}}\right) \mathcal{A}_{2}^{-1} w_{2}-\mathrm{Pe}_{\text {mic }}((\mathbf{u}-\mathbf{v}) \cdot \nabla) \mathcal{A}_{2}^{-1} w_{1}\right\} d \mathbf{y}=0 .
\end{aligned}
$$

The term multiplied by $\lambda$ in (38) can immediately be rewritten by

$$
\lambda \int_{Y^{1}}-\left(\mathcal{B}_{2} w_{0}+\mathcal{B}_{1} w_{1}\right) d \mathbf{y}=-\lambda \operatorname{div}\left(\hat{\mathrm{M}}_{w} \nabla w_{0}\right)
$$

where the effective tensor $\hat{M}_{w}=\left\{\mathrm{m}_{i k}^{w}\right\}_{1 \leq i, k \leq d}$ is defined by

$$
\mathrm{m}_{i k}^{w}:=\frac{1}{|Y|} \sum_{j=1}^{d} \int_{Y^{1}}\left(\mathrm{~m}_{i k}-\mathrm{m}_{i j} \frac{\partial \xi_{w}^{k}}{\partial y_{j}}\right) d \mathbf{y} .
$$

The first term on the second line in (38) transform as in [42] to

$$
\begin{aligned}
-\mathcal{B}_{1} & {\left[f^{\prime}\left(\phi_{0}\right) \phi_{1}\right] } \\
& =-\sum_{i, j=1}^{d}\left[\frac{\partial}{\partial x_{i}}\left(\mathrm{~m}_{i j} f^{\prime}\left(\phi_{0}\right) \sum_{k=1}^{d} \frac{\partial \xi_{\phi}^{k}}{\partial y_{j}} \frac{\partial \phi_{0}}{\partial x_{k}}\right)+\frac{\partial}{\partial y_{i}}\left(\mathrm{~m}_{i j} f^{\prime}\left(\phi_{0}\right) \sum_{k=1}^{d} \xi_{\phi}^{k} \frac{\partial^{2} \phi_{0}}{\partial x_{k} \partial x_{j}}\right)\right]
\end{aligned}
$$

where the last term in (41) disappears after integrating by parts. The first term on the right-hand side of (41) can be rewritten with the help of the chain rule as follows

$$
-\mathcal{B}_{1}\left[f^{\prime}\left(\phi_{0}\right) \phi_{1}\right]=-\sum_{i, j=1}^{d} \mathrm{~m}_{i j} \sum_{k=1}^{d} \frac{\partial \xi_{\phi}^{k}}{\partial y_{j}} \frac{\partial^{2} f\left(\phi_{0}\right)}{\partial x_{k} \partial x_{i}}
$$

After adding to 42 the term $-\mathcal{B}_{2} f\left(\phi_{0}\right)$, we can define a tensor $\hat{\mathrm{M}}_{\phi}=\left\{\mathrm{m}_{i j}^{\phi}\right\}_{1 \leq i, k \leq d}$, i.e.,

$$
\mathrm{m}_{i k}^{\phi}:=\frac{1}{|Y|} \sum_{j=1}^{d} \int_{Y^{1}}\left(\mathrm{~m}_{i k}-\mathrm{m}_{i j} \frac{\partial \xi_{\phi}^{k}}{\partial y_{j}}\right) d \mathbf{y}
$$


such that

$$
-\mathcal{B}_{1}\left[f^{\prime}\left(\phi_{0}\right) \phi_{1}\right]-\mathcal{B}_{2} f\left(\phi_{0}\right)=\operatorname{div}\left(\hat{M}_{\phi} \nabla f\left(\phi_{0}\right)\right) .
$$

The terms in the last line of (38) become

$$
\begin{aligned}
-\frac{1}{|Y|} \int_{Y^{1}} & \operatorname{Pe}_{m i c}\left(\mathbf{u} \cdot \nabla_{\mathbf{y}}\right) \mathcal{A}_{2}^{-1} w_{2}+\operatorname{Pe}_{m i c}((\mathbf{u}-\mathbf{v}) \cdot \nabla) \mathcal{A}_{2}^{-1} w_{1} d \mathbf{y} \\
= & -\frac{1}{|Y|} \int_{Y^{1}} \operatorname{Pe}_{m i c}\left(\mathbf{u} \cdot \nabla_{\mathbf{y}}\right) \phi_{2} d \mathbf{y} \\
& +\frac{\mathrm{Pe}_{m i c}}{|Y|} \sum_{k, i=1}^{d} \int_{Y^{1}}\left(\mathrm{u}^{i}-\mathrm{v}^{i}\right) \frac{\partial}{\partial x_{i}}\left(\delta_{i k} \xi_{\phi}^{k}(\mathbf{y}) \frac{\partial}{\partial x_{k}} \phi_{0}\right) d \mathbf{y} .
\end{aligned}
$$

Using the fact that $\mathbf{u}$ is divergence-free and after defining the tensor $\hat{\mathrm{C}}:=\left\{\mathrm{c}_{i k}\right\}_{1 \leq i, k \leq d}$ by

$$
\mathrm{c}_{i k}:=\frac{\mathrm{Pe}_{m i c}}{|Y|} \int_{Y^{1}}\left(\mathrm{u}^{i}-\mathrm{v}^{i}\right) \delta_{i k} \xi_{\phi}^{k}(\mathbf{y}) d \mathbf{y}
$$

we finally obtain with the previous considerations the following upscaled phase field equation

$$
\begin{aligned}
p \frac{\partial \mathcal{A}_{2}^{-1} w_{0}}{\partial t}= & \operatorname{div}\left(\left[\lambda \hat{\mathrm{M}}_{\phi} f^{\prime}\left(\phi_{0}\right)+\hat{\mathrm{C}}\right] \nabla \phi_{0}\right) \\
& -\frac{\lambda}{p} \operatorname{div}\left(\hat{\mathrm{M}}_{w} \nabla\left(\operatorname{div}\left(\hat{\mathrm{D}} \nabla \phi_{0}\right)-\tilde{g}_{0}\right)\right) .
\end{aligned}
$$

Using (36) finally leads to the effective macroscopic phase field equation

$$
\begin{aligned}
p \frac{\partial \phi_{0}}{\partial t}= & \operatorname{div}\left(\left[\lambda \hat{\mathrm{M}}_{\phi} f^{\prime}\left(\phi_{0}\right)+\hat{\mathrm{C}}\right] \nabla \phi_{0}\right) \\
& -\frac{\lambda}{p} \operatorname{div}\left(\hat{\mathrm{M}}_{w} \nabla\left(\operatorname{div}\left(\hat{\mathrm{D}} \nabla \phi_{0}\right)-\tilde{g}_{0}\right)\right) .
\end{aligned}
$$

\section{Proof of Theorem 3.5}

The proof follows in three basic steps: In Step 1, we establish a priori estimates that provide compactness required for Step 2 where we construct a sequence of approximate solutions. In Step 3, we pass to the limit in the sequence of approximate solutions which provide then existence and uniqueness of the original problem.

Step 1: (A priori estimates) i) Basic energy estimate: As we establish the existence and uniqueness of weak solutions, we rewrite (23) in the sense of distributions, i.e., for all $\varphi \in H_{E}^{2}$ it holds that

$$
\begin{aligned}
\frac{p}{2} \frac{\partial}{\partial t}\left(\phi_{0}, \varphi\right) & +\frac{\lambda}{p}\left(\operatorname{div}\left(\hat{\mathrm{D}} \nabla \phi_{0}\right), \operatorname{div}\left(\hat{\mathrm{M}}_{w} \nabla \varphi\right)\right)+\frac{\lambda}{p}\left(\hat{\mathrm{M}}_{w} \nabla \tilde{g}_{0}, \nabla \varphi\right) \\
& +\left(\lambda f^{\prime}\left(\phi_{0}\right) \hat{\mathrm{M}}_{\phi} \nabla \phi_{0}, \nabla \varphi\right)+\left(\hat{\mathrm{C}} \nabla \phi_{0}, \nabla \varphi\right)=0 .
\end{aligned}
$$


Note that for an isotropic mobility of the form $\hat{\mathrm{M}}=m \hat{\mathrm{I}}$, where $\hat{\mathrm{I}}$ is the identity tensor, the following identity holds $m \hat{\mathrm{D}}=\hat{\mathrm{M}}_{w}$. For simplicity, we will base our proof on this identity. The general case is then verified along the same lines using properties of symmetric positive definite tensors. Using the notation $\Delta_{\hat{\mathrm{D}}}:=\operatorname{div}(\hat{\mathrm{D}} \nabla)$ and the test function $\varphi=\phi_{0}$ leads to the following inequality

$$
\begin{aligned}
\frac{p}{2} \frac{d}{d t}\left\|\phi_{0}\right\|^{2} & +\frac{\lambda m}{p}\left\|\Delta_{\hat{\mathrm{D}}} \phi_{0}\right\|^{2}+\lambda b_{2 r} m_{\phi} \int_{\Omega} \phi_{0}^{2 r-2}\left|\nabla \phi_{0}\right|^{2} d \mathbf{x} \\
& \leq C\left\|\nabla \phi_{0}\right\|^{2}+\frac{2 \lambda m_{w}}{p}\left\|\nabla \tilde{g}_{0}\right\|\left\|\nabla \phi_{0}\right\| \leq C\left\|\nabla \phi_{0}\right\|^{2}+\frac{\lambda}{2 p} m_{w}\left\|\nabla \tilde{g}_{0}\right\|^{2},
\end{aligned}
$$

where we applied the existence of a constant $C>0$ such that $f(s) s \geq p b_{2 r} s^{2 r}-C$, for all $s \in \mathbb{R}$ due to Assumption (PF). With the equivalence of norms in $H^{2}(\Omega)$, i.e., $\left\|\phi_{0}\right\|_{H^{2}(\Omega)} \leq C\left(\left\|\Delta \phi_{0}\right\|+\left\|m\left(\phi_{0}\right)\right\|\right)$, and interpolation estimates we obtain

$$
\begin{aligned}
\left\|\nabla \phi_{0}\right\|^{2} & \leq C\left\|\phi_{0}\right\|\left\|\phi_{0}\right\|_{H^{2}(\Omega)} \leq C\left\|\phi_{0}\right\|\left(\left\|\Delta \phi_{0}\right\|+\alpha\right) \leq C\left\|\phi_{0}\right\|\left(\left\|\Delta_{\hat{\mathrm{D}}} \phi_{0}\right\|+\alpha\right) \\
& \leq \frac{\kappa}{2}\left\|\Delta_{\hat{\mathrm{D}}} \phi_{0}\right\|^{2}+\frac{1}{C \kappa}\left\|\phi_{0}\right\|^{2}+\frac{\kappa \alpha^{2}}{2},
\end{aligned}
$$

where $m\left(\phi_{0}\right):=\frac{1}{|\Omega|} \int_{\Omega} \phi_{0} d \mathbf{x}$ with $m\left(\phi_{0}\right) \leq \alpha$. This leads to

$$
\begin{aligned}
\frac{p}{2} \frac{d}{d t}\left\|\phi_{0}\right\|^{2} & +\left(\frac{\lambda m}{p}-\frac{\kappa}{2}\right)\left\|\Delta \phi_{0}\right\|^{2}+\lambda b_{2 r} \int_{\Omega} \phi^{2 r-2}\left|\nabla \phi_{0}\right|^{2} d \mathbf{x} \\
& \leq C(\kappa)\left\|\phi_{0}\right\|^{2}+C(\alpha, \kappa)+\frac{\lambda}{2 p} m_{w}\left\|\nabla \tilde{g}_{0}\right\|^{2},
\end{aligned}
$$

which turns with Gronwall's inequality into the expression

$$
\left\|\phi_{0}(\cdot, t)\right\|^{2} \leq\left\|\phi_{0}(\cdot, 0)\right\|^{2} \exp (C t)+\int_{0}^{t}\left(C(\alpha)+\frac{\lambda}{2 p} m_{w}\left\|\nabla \tilde{g}_{0}\right\|^{2}\right) \exp (-C s) d s,
$$

for $T^{*} \geq 0$ such that we finally obtain $\phi \in L^{\infty}\left(0, T^{*} ; L^{2}(\Omega)\right) \cap L^{2}\left(0, T^{*} ; H_{E}^{2}(\Omega)\right)$.

ii) Control over time derivative: Using the test function $\varphi \in H_{E}^{2}(\Omega)$ in $(49)$ allows us to estimate the time derivative term by

$$
\frac{p}{2}\left|\left(\partial_{t} \phi_{0}, \varphi\right)\right| \leq C\left(\left\|\Delta_{\hat{\mathrm{D}}} \phi_{0}\right\|+\left\|\tilde{g}_{0}\right\|+\left\|f\left(\phi_{0}\right)\right\|+\left\|\phi_{0}\right\|\right)\|\Delta \varphi\|
$$

such that $\left\|\partial_{t} \phi_{0}\right\|_{\left(H_{E}^{2}(\Omega)\right)^{*}}=\sup _{\varphi \in H_{E}^{2}} \frac{\left|\left(\partial_{t} \phi_{0}, \varphi\right)\right|}{\|\varphi\|_{H_{E}^{2}}} \leq C$ and hence $\left\|\partial_{t} \phi_{0}\right\|_{\left(H_{E}^{2}(\Omega)\right)^{*}}^{2} \leq C\left(T^{*}\right)$.

Step 2: (Galerkin approximation) As $H_{E}^{2}(\Omega)$ is a separable Hilbert space, we can identify a linearly independent basis $\varphi_{j} \in H_{E}^{2}(\Omega), j \in \mathbb{N}$, which is complete in $H_{E}^{2}(\Omega)$. This allows us to define for each $N \in \mathbb{N}$ approximate solutions $\phi_{0}^{N}=\sum_{j=1}^{N} \eta_{j}^{N}(t) \varphi_{j}$ which solve

$$
\begin{aligned}
\frac{p}{2} \frac{\partial}{\partial t}\left(\phi_{0}^{N}, \varphi_{j}\right) & +\frac{\lambda m}{p}\left(\operatorname{div}\left(\hat{\mathrm{D}} \nabla \phi_{0}^{N}\right), \operatorname{div}\left(\hat{\mathrm{D}} \nabla \varphi_{j}\right)\right)+\frac{\lambda}{p}\left(\hat{\mathrm{M}}_{w} \nabla \tilde{g}_{0}, \nabla \varphi_{j}\right) \\
& +\left(\lambda f^{\prime}\left(\phi_{0}^{N}\right) \hat{\mathrm{M}}_{\phi} \nabla \phi_{0}^{N}, \nabla \varphi_{j}\right)+\left(\hat{\mathrm{C}} \nabla \phi_{0}^{N}, \nabla \varphi_{j}\right)=0 \quad j=1, \ldots, N
\end{aligned}
$$


for the initial condition $\phi_{0}^{N}(\cdot, 0)=\psi^{N}(\cdot)$. The initial value problem 55 represents a system of $N$ ordinary differential equations (ODEs) for the coefficients $\eta_{j}^{N}(t)$. Hence, classical ODE theory immediately provides existence and uniqueness of $\phi_{0}^{N}$. Moreover, we have $\phi_{0}^{N} \in C\left(0, T^{*} ; H_{E}^{2}(\Omega)\right)$ and $\partial_{t} \phi_{0}^{N} \in L^{2}\left(0, T^{*} ; H_{E}^{-2}(\Omega)\right)$.

Step 3: (Passing to the limit) In the same way as in Step 1, we can derive a priori estimates for the approximate solutions $\phi_{0}^{N}$ by using the test function $\phi_{0}^{N}$ instead of the basis functions $\varphi_{j}$ in (55). Hence, by weak compactness, see Theorem 2.1 in Section 2 , there exist subsequences (for simplicity still denoted by $\phi_{0}^{N}$ ) such that

$$
\begin{array}{ll}
\phi_{0}^{N} \rightarrow \phi_{0} & \text { in } L^{2}\left(0, T^{*} ; H_{E}^{2}(\Omega)\right) \text { weakly, } \\
\phi_{0}^{N} \stackrel{*}{\rightarrow} \phi_{0} & \text { in } L^{\infty}\left(0, T^{*} ; L^{2}(\Omega)\right) \text { weak-star }, \\
\phi_{0}^{N} \rightarrow \phi_{0} & \text { in } L^{2}\left(0, T^{*} ; L^{2}(\Omega)\right) \text { strongly, }
\end{array}
$$

for $N \rightarrow \infty$. This allows us to pass to the limit in the initial value problem (55) such that we obtain

$$
\begin{aligned}
\frac{p}{2} \frac{\partial}{\partial t}\left(\phi_{0}, \varphi\right) & +\frac{\lambda}{p}\left(\operatorname{div}\left(\hat{\mathrm{D}} \nabla \phi_{0}\right), \operatorname{div}(m \hat{\mathrm{D}} \nabla \varphi)\right)+\frac{\lambda}{p}\left(\hat{\mathrm{M}}_{w} \nabla \tilde{g}_{0}, \nabla \varphi\right) \\
& +\left(\lambda f^{\prime}\left(\phi_{0}\right) \hat{\mathrm{M}}_{\phi} \nabla \phi_{0}, \nabla \varphi\right)+\left(\hat{\mathrm{C}} \nabla \phi_{0}, \nabla \varphi\right)=0,
\end{aligned}
$$

for all $\varphi \in H_{E}^{2}(\Omega)$. In the same way we can pass to the limit with respect to the initial condition $\psi^{N}$.

\section{Conclusions}

The main new result here is the extension of the the study by Schmuck et al. in the absence of flow [42] to include a periodic fluid flow in the case of sufficiently large Péclet number. The resulting new effective porous media approximation (19) of the microscopic Stokes-Cahn-Hilliard problem (11)-(12) reveals interesting physical characteristics such as diffusion-dispersion relations by $(20)_{2}-(20)_{3}$ for instance. The homogenization methodology developed here allows for the systematic and rigorous derivation of effective macroscopic porous media equations as motivated for instance in [18] where the author identifies Darcy's law after upscaling the Stokes equations. By a slight modification of the homogenization method as initiated in [3, 10, 27] for diffusionconvection problems, one recovers here for the first time, to the best knowledge of the authors, rigorous and systematic diffusion-dispersion relations for general Stokes-CahnHilliard equations.

We note that the Cahn-Hilliard and related equations generally model more complex material transport [29, 35] than classical Fickian diffusion. In this context, our result of an upscaled convective Cahn-Hilliard equation hence provides diffusion-dispersion relations for generalized non-Fickian material transport, that is, not just the product of a gradient of particle concentration and a constant diffusion matrix. 
Our current periodicity assumption on the fluid flow imposed by (11) implies a quasi-steady state on the fluid velocity and seems inevitable for the homogenization theory to work such as the assumption of a scale separated chemical potential on the level of the reference cells.

So far, we are restricted to mixtures of two fluids by our model. An extension towards mixtures of $N>2$ components is studied in [30] where the authors compare a local and non-local model for incompressible fluids. In specific applications, it might be of interest to extend the here developed framework towards such multi-component mixtures.

Finally, the rigorous and systematic derivation of effective macroscopic immiscible flow equations provides a promising and convenient alternative in view of the broad applicability of the Cahn-Hilliard equations. The strength of our approach is based on its foundation on a thermodynamically motivated homogeneous free energy which is generally derived on systematic physical grounds. Moreover, even if a systematic derivation is impossible, one can mathematically design such a free energy based on physical principles and phenomenological observations. As an example of the applicability of the presented upscaled equations, we have numerically solved the homogenised Cahn-Hilliard equation for a porous medium defined by periodic reference cells consisting of a characteristic perturbed straight channel. We observe that the macroscopic solution retained the geometric characteristics induced at the microscopic scale. This numerical study represents a first step towards the use of the presented methodology in more complex geometries with e.g. non-periodic properties, something that we leave as a future work. In addition, a more detailed numerical study shall allow to rationally analyze current applications in science and engineering and hopefully reveal potential new ones.

\section{Acknowledgements}

We acknowledge financial support from EPSRC Grant No. EP/H034587, EPSRC Grant No. EP/J009636/1, EU-FP7 ITN Multiflow and ERC Advanced Grant No. 247031.

\section{References}

[1] H. Abels. Double obstacle limit for a Navier-Stokes/Cahn-Hilliard system. in Progress in Nonlinear Differential Equations and Their Applications, 43:1-20, Springer 2011.

[2] P. M. Adler and H. Brenner. Multiphase flow in porous media. Ann. Rev. Fluid Mech., 20:35-39, 1988.

[3] G. Allaire, R. Brizzi, A. Mikelić, and A. Piatnitski. Two-scale expansion with drift approach to the Taylor dispersion for reactive transport through porous media. Chem. Eng. Sci., 65:2292-2300, 2010. 
[4] R. Aris. On the Dispersion of a Solute in a Fluid Flowing through a Tube. Proc. R. Soc. A, 235(1200):67-77, 1956 doi: 10.2307/100013. URL http: //dx.doi.org/10.2307/100013.

[5] R. J. Atkin and R. E. Craine. Continuum theories of mixtures: Applications. J. Inst. Math. Appl., 17(2):153-207, 1976. ISSN 0020-2932.

[6] J.-L. Auriault and J. Lewandowska. Effective Diffusion Coefficient: From Homogenization to Experiment. Transp. Porous Med., 27(2):205-223, 1997. ISSN 0272-4960 (print), 1464-3634 (electronic). URL http://www.springerlink.com/ content/r68u773353484u38/.

[7] A. Bensoussan, J.-L. Lions, and G. Papanicolaou. Analysis for Periodic Structures. North-Holland Publishing Company, North-Holland, Amsterdam, 1978.

[8] A. Bourgeat, S. Luckhaus, and A. Mikelić. Convergence of the homogenization process for a double-porosity model of immiscible two-phase flow. SIAM Journal on Mathematical Analysis, 27(6):1520-1543, 1996. doi: 10.1137/S0036141094276457. URL http://epubs.siam.org/doi/abs/10.1137/S0036141094276457.

[9] F. Boyer. A theoretical and numerical model for the study of incompressible mixture flows. Comput. Fluids, 31(1):41 - 68, 2002. ISSN 0045-7930. doi: 10. 1016/S0045-7930(00)00031-1. URL http://www.sciencedirect.com/science/ article/pii/S0045793000000311.

[10] H. Brenner. Dispersion Resulting from Flow through Spatially Periodic Porous Media. Phil. Trans. Roy. Soc. London, A, 297(1430):81-133, July 1980. doi: 10.1098/rsta.1980.0205. URL http://dx.doi.org/10.1098/rsta.1980.0205.

[11] R.G. Carbonell and S. Whitaker. Dispersion in pulsed systems - II: Theoretical developments for passive dispersion in porous media. Chem. Engng Sci., 38(11): 1795 - 1802, 1983. ISSN 0009-2509. doi: 10.1016/0009-2509(83)85036-2. URL http://www. sciencedirect.com/science/article/pii/0009250983850362.

[12] P. M. Chaikin and T. C. Lubensky. Principles of Condensed Matter Physics. Cambridge University Press, Cambridge, 1995.

[13] D. Cioranescu and P. Donato. An Introduction to Homogenization, volume 17 of Oxford Lecture Series in Mathematics and its Applications. Oxford University Press, New York, 1999. ISBN 0-19-856554-2.

[14] P. G. de Gennes and R. L. Prost. The Physics of Liquid Crystals. Oxford University Press, 1993.

[15] M. Doi. The Theory of Polymer Dynamics. Oxford Science Publication, 1986.

[16] C. Eck, M. Fontelos, G. Grün, F. Klingbeil, and O. Vantzos. On a phase-field model for electrowetting. Interface Free Bound., 11(2):259-290, 2009. ISSN 1463-9963. doi: 10.4171/IFB/211. URL http://www.ems-ph.org/doi/10.4171/IFB/211.

[17] J. J. Feng, C. Liu, J. Shen, and P. Yue. An energetic variational formulation with phase field methods for interfacial dynamics of complex fluids: advantages and challenges. IMA Volumes in Mathematics and Applications, 140:1-26, 2005. 
[18] U. Hornung. Homogenization and Porous Media. Springer, 1997.

[19] Y. Hyon, D. Y. Kwak, and C. Liu. Energetic variational approach in complex fluids: Maximum dissipation principle. Discrete Cont. Dyn. S., 26(4):1291-1304, 2010. ISSN 1078-0947. doi: 10.3934/dcds.2010.26.1291. URL http://www . aimsciences . org/journals/displayArticles . jsp?paper ID=4774.

[20] P. Jenny, S. H. Lee, and H. A. Tchelepi. Multi-scale finite-volume method for elliptic problems in subsurface flow simulation. Journal of Computational Physics, 187(1):47 - 67, 2003. ISSN 0021-9991. doi: http://dx.doi.org/10. 1016/S0021-9991(03)00075-5. URL http://www.sciencedirect.com/science/ article/pii/S0021999103000755.

[21] F. H. Lin and C. Liu. Nonparabolic dissipative systems, modeling the flow of liquid crystals. Comm. Pure Appl. Math., XLVIII:501-537, 1995.

[22] C. Liu and J. Shen. A phase field model for the mixture of two incompressible fluids and its approximation by a fourier-spectral method. Phys. D, 179:211-228, 2003.

[23] C. Liu and N. J. Walkington. An Eulerian description of fluids containing viscohyperelastic particles. Arch. Rat. Mech. Anal., 159:229-252, 2001.

[24] J. Lowengrub and L. Truskinovsky. Quasi-incompressible Cahn-Hilliard fluids and topological transitions. Proc. R. Soc. A, 454:2617-2654, 1998.

[25] H.-W. Lu, K. Glasner, A. L. Bertozzi, and C.-J. Kim. A diffuse-interface model for electrowetting drops in a hele-shaw cell. J. Fluid Mech., 590:411-435, 11 2007. ISSN 1469-7645. doi: 10.1017/S0022112007008154. URL http://journals . cambridge. org/article_S0022112007008154.

[26] C. M. Marle. On macroscopic equations governing multiphase flow with diffusion and chemical reactions in porous media. Int. J. Eng. Sci., 20:643-662, 1982.

[27] E. Marušic-Paloka and A. L. Piatnitski. Homogenization of a nonlinear convectiondiffusion equation with rapidly oscillating coefficients and strong convection. $J$. London Math. Soc., 72(02):391, October 2005. ISSN 0024-6107. doi: 10. 1112/S0024610705006824. URL http://jlms.oxfordjournals.org/cgi/doi/ 10.1112/S0024610705006824.

[28] C. C. Mei. Method of homogenization applied to dispersion in porous media. Transp. Porous Media, 9:261-274, 1992. ISSN 0169-3913. URL http://dx.doi. org/10.1007/BF00611970. 10.1007/BF00611970.

[29] A. Novick-Cohen. The Cahn-Hilliard equation. In Handbook of differential equations: evolutionary equations. Vol. IV, Handb. Differ. Equ., pages 201-228. Elsevier/North-Holland, Amsterdam, 2008. doi: 10.1016/S1874-5717(08)00004-2. URL http://dx.doi .org/10.1016/S1874-5717(08)00004-2.

[30] F. Otto and W. E. Thermodynamically driven incompressible fluid mixtures. J. Chem. Phys., 107(23):10177-10184, 1997. doi: 10.1063/1.474153. URL http: //link.aip.org/link/?JCP/107/10177/1. 
[31] P. Papatzacos. Macroscopic two-phase flow in porous media assuming the diffuseinterface model at pore level. Transp. Porous Med., 49:139-174, 2002.

[32] P. Papatzacos. A model for multiphase and multicomponent flow in porous media, built on the diffuse interface assumption. Transp. Porous Med., 82:443-462, 2010.

[33] G. A. Pavliotis and A. M. Stuart. Multiscale Methods, volume 53 of Texts in Applied Mathematics. Springer, New York, 2008. ISBN 978-0-387-73828-4. Averaging and Homogenization.

[34] R. F. Probstein. Physicochemical Hydrodynamics: An Introduction. Wiley, New York, 1994.

[35] K. Promislow and B. Wetton. PEM fuel cells: a mathematical overview. SIAM J. Appl. Math., 70(2):369-409, 2009. ISSN 0036-1399. doi: 10.1137/080720802. URL http://dx.doi.org/10.1137/080720802.

[36] M. Quintard and S. Whitaker. Transport in ordered and disordered porous media: volume-averaged equations, closure problems, and comparison with experiment. Chem. Engng Sci., 48(14):2537-2564, July 1993. ISSN 00092509. doi: 10. 1016/0009-2509(93)80266-S. URL http://dx.doi.org/10.1016/0009-2509(93) 80266-S.

[37] J. Rubinstein and R. Mauri. Dispersion and convection in periodic porous media. SIAM J. Appl. Math., 46(6):1018-1023, 1986. ISSN 00361399. URL http://www.jstor.org/stable/2101656.

[38] T. Savin, K. S. Glavatskiy, S. Kjelstrup, H. C. Öttinger, and D. Bedeaux. Local equilibrium of the Gibbs interface in two-phase systems. EPL (Europhysics Letters), 97(4):40002, 2012. URL http://stacks.iop.org/0295-5075/97/i=4/a=40002.

[39] M. Schmuck. First error bounds for the porous media approximation of the PoissonNernst-Planck equations. ZAMM-Z. Angew. Math. Me., 92(4):304-319, 2012. ISSN 00442267. doi: 10.1002/zamm.201100003. URL http://doi.wiley.com/10.1002/ zamm. 201100003.

[40] M. Schmuck. A new upscaled Poisson-Nernst-Planck system for strongly oscillating potentials. J. Math. Phys., 54:021504, 2013.

[41] M. Schmuck and P. Berg. Homogenization of a Catalyst Layer Model for Periodically Distributed Pore Geometries in PEM Fuel Cells. Appl. Math. Res. Express., 2013(1):57-78, July 2013. ISSN 1687-1200. doi: 10.1093/amrx/abs011. URL http://amrx . oxfordjournals .org/cgi/doi/10.1093/amrx/abs011.

[42] M. Schmuck, M. Pradas, G. A. Pavliotis, and S. Kalliadasis. Upscaled phase-field models for interfacial dynamics in strongly heterogeneous domains. Proc. R. Soc. A, 468:3705-3724, 2012. doi: doi:10.1098/rspa.2012.0020.

[43] B. Schweizer. Homogenization of degenerate two-phase flow equations with oil trapping. SIAM Journal on Mathematical Analysis, 39(6):1740-1763, 2008. doi: 10. 1137/060675472. URL http://epubs .siam.org/doi/abs/10.1137/060675472. 
[44] R. E. Showalter. Monotone operators in Banach space and nonlinear partial differential equations, volume 49 of Mathematical Surveys and Monographs. American Mathematical Society, Providence, RI, 1997. ISBN 0-8218-0500-2.

[45] D.N. Sibley, A. Nold, N. Savva, and S. Kalliadasis. On the moving contact line singularity: Asymptotics of a diffuse-interface model. Eur. Phys. J. E, 36:26, 2013.

[46] D.N. Sibley, A. Nold, N. Savva, and S. Kalliadasis. The contact line behaviour of solid-liquid-gas diffuse-interface models. Phys. Fluids, 25:092111, 2013.

[47] M. Struwe. Variational Methods: Applications to Nonlinear Partial Differential Equations and Hamiltonian Systems. Springer, Dordrecht, 2008.

[48] G.I. Taylor. Dispersion of Soluble Matter in Solvent Flowing Slowly through a Tube. Proc. R. Soc. A, 219(1137):186-203, August 1953. doi: 10.1098/rspa.1953.0139. URL http://dx.doi.org/10.1098/rspa.1953.0139.

[49] R. Temam. Infinite Dimensonal Dynamical Systems in Mechanics and Physics. Applied Mathematical Sciences. Springer, 2nd edition, 1997. ISBN 9780387948669.

[50] S. Whitaker. Flow in porous media I: A theoretical derivation of Darcy's law. Transport Porous Med., 1:3-25, 1986. ISSN 0169-3913. URL http://dx.doi.org/ 10.1007/BF01036523. 10.1007/BF01036523.

[51] C. Wylock, M. Pradas, B. Haut, C. Colinet, and S. Kalliadasis. Disorder-induced hysteresis and nonlocality of contact line motion in chemically heterogeneous microchannels. Phys. Fluids, 24:032108, 2012. 